\title{
Metabolic and microstructural alterations in the SLE brain correlate with cognitive impairment
}

\author{
Meggan Mackay, ${ }^{1}$ An Vo, ${ }^{2}$ Chris C. Tang, ${ }^{2}$ Michael Small, ${ }^{2}$ Erik W. Anderson, ${ }^{1}$ Elisabeth J. Ploran, ${ }^{3}$ \\ Justin Storbeck, ${ }^{4}$ Brittany Bascetta, ${ }^{4}$ Simran Kang, ${ }^{4}$ Cynthia Aranow, ${ }^{1}$ Carl Sartori, ${ }^{1}$ Philip Watson, ${ }^{5}$ \\ Bruce T. Volpe, ${ }^{1}$ Betty Diamond, ${ }^{1}$ and David Eidelberg ${ }^{2}$ \\ 1Autoimmune, Musculoskeletal and Hematopoietic Diseases and ${ }^{2}$ Center for Neurosciences, Feinstein Institute for \\ Medical Research and Donald and Barbara Zucker School of Medicine at Hofstra/Northwell, Manhasset, New York, USA. \\ ${ }^{3}$ Department of Psychology, Hofstra University, Hempstead, New York, USA. ${ }^{4}$ Department of Psychology, Queens College, \\ Flushing, New York, USA. ${ }^{5}$ Department of Psychiatry, Northwell Health, Manhasset, New York, USA.
}

\begin{abstract}
To address challenges in the diagnosis of cognitive dysfunction (CD) related to systemic lupus erythematosus-associated (SLE-associated) autoimmune mechanisms rather than confounding factors, we employed an integrated approach, using resting-state functional (FDG-PET) and structural (diffusion tensor imaging [DTI]) neuroimaging techniques and cognitive testing, in adult SLE patients with quiescent disease and no history of neuropsychiatric illness. We identified resting hypermetabolism in the sensorimotor cortex, occipital lobe, and temporal lobe of SLE subjects, in addition to validation of previously published resting hypermetabolism in the hippocampus, orbitofrontal cortex, and putamen/GP/thalamus. Regional hypermetabolism demonstrated abnormal interregional metabolic correlations, associated with impaired cognitive performance, and was stable over 15 months. DTI analyses demonstrated 4 clusters of decreased microstructural integrity in white matter tracts adjacent to hypermetabolic regions and significantly diminished connecting tracts in SLE subjects. Decreased microstructural integrityin the parahippocampal gyrus correlated with impaired spatial memory and increased serum titers of DNRAb, a neurotoxic autoantibody associated with neuropsychiatric lupus. These findings of regional hypermetabolism, associated with decreased microstructural integrity and poor cognitive performance and not associated with disease duration, disease activity, medications, or comorbid disease, suggest that this is a reproducible, stable marker for SLE-associated CD that may be may be used for early disease detection and to discriminate between groups, evaluate response to treatment strategies, or assess disease progression.
\end{abstract}

Authorship note: MM, AV, and CCT are co-first authors.

Conflict of interest: The authors have declared that no conflict of interest exists.

License: Copyright 2019, American Society for Clinical Investigation.

Submitted: August 3, 2018

Accepted: December 5, 2018

Published: January 10, 2019

Reference information:

JCI Insight. 2019;4(1):e124002.

https://doi.org/10.1172/jici.

insight.124002.

\section{Introduction}

Cognitive dysfunction (CD) is one of the most common of the 19 neuropsychiatric lupus (NPSLE) syndromes and manifests insidiously over time, as opposed to the more dramatic presentations of acute confusional state, stroke, and psychosis $(1,2) . C D$ in systemic lupus erythematosus (SLE) affects multiple cognitive domains, including attention, executive function, verbal and nonverbal learning, working memory, and psychomotor function; it is associated with significantly increased morbidity and decreased quality of life (3-6). The diagnosis of CD and correct attribution to SLE-associated mechanisms rather than other potentially confounding factors, such as infection, metabolic disturbances, medication effects, mood disturbances, and toxins, have been compromised by the poor sensitivity and specificity of available diagnostic tools. Additionally, the wide variability of CD prevalence in lupus, ranging from $3 \%$ to $80 \%$, is ascribed to differences in testing metrics, subject demographics and comorbidities, and small sample sizes (7). These conflicting reports highlight the need for unbiased and objective assessments of, or biomarkers for, CD attributable to SLE that can be used for diagnosis and assessment of response to treatment.

Proposed mechanisms for $\mathrm{CD}$ include chronic neurotoxic effects of brain-reactive autoantibodies directed against $\mathrm{N}$-methyl $\mathrm{D}$ aspartate receptors (cross-reactive anti-dsDNA/NMDAR antibodies, termed DNRAb) $(8,9)$, ribosomal $P$, and neuronal surface $P$ antigen and direct effects of cytokines (10-13). In a 
murine model, DNRAb bind activated NMDARs, allowing excessive calcium influx and resulting in neuronal dysfunction or death, depending on antibody concentration (14). NMDARs are located throughout the cortex but have highest density in the hippocampus; it has also been shown that DNRAb specifically target place cells in the CA-1 area of the hippocampus, with resulting deleterious effects on spatial memory in mice and human disease $(14,15)$.

Fluorine-18 fluorodeoxyglucose PET ([18F]FDG-PET) is a functional imaging technique that measures cellular glucose uptake. Alterations in CNS glucose metabolism may reflect cell density, infection, inflammation, and/or abnormal cell function. We have reported previously that SLE subjects with stable disease activity and without known CNS disease demonstrate a pattern of increased resting metabolism in the hippocampus, orbitofrontal cortex, and basal ganglia (16) that correlated with serum DNRAb titers, memory impairment, and mood alterations. A complementary neuroimaging technique, diffusion tensor imaging (DTI), is an advanced MRI technique based on the diffusion of water molecules in tissue that allows for assessment of white matter (WM) integrity and structure. Several cross-sectional studies of DTI in NPSLE and SLE patients without neuropsychiatric symptoms, compared with healthy controls, suggest an overall decrease in WM integrity in both SLE groups (reviewed in ref. 17). However, combining imaging results for all NPSLE syndromes precludes the ability to evaluate individual CNS manifestations in the context of proposed biologic mechanisms.

We therefore sought to continue our neuroimaging characterization of CD in SLE and visualize structural and metabolic changes that might embody a biomarker for SLE-associated pathophysiological processes. Based on our previously reported set of regional metabolism changes in SLE that correlated with neuropsychiatric performance (16), we (a) validated those original findings in a new cohort and a larger combined cohort; (b) defined changes in underlying tract microstructure in the new cohort; (c) assessed longitudinal changes in brain metabolism and microstructure in these individuals; and (d) related the observed changes to cognitive performance and other variables, such as disease duration and serum DNRAb levels. We continue to focus on SLE patients with no known history of an acute clinical NPSLE manifestation or other CNS event in order to avoid confounding influences from other NPSLE-related or unrelated syndromes that may be driven by different underlying mechanisms.

\section{Results}

\section{Clinical characteristics of SLE groups 1 and 2}

Thirty-seven adult SLE subjects were recruited. Data from the first 17 SLE subjects (group 1 [SLE-1]) were published previously (16). Both groups were similar for all measured parameters, except for anti-Ro antibodies, which were less frequent in SLE-1 (Table 1), and disease duration. The SLE-1 group had been stratified by disease duration of less than or equal to 2 years $(n=9)$ or greater than or equal to 10 years $(n=8)$. The mean disease duration in those with disease for longer than or equal to 10 years was $16.25 \pm 6.78$ years (range 10-28 years). Because regional brain hypermetabolism did not correlate with disease duration in our previous study (16), SLE-2 subjects were not stratified by disease duration. The mean disease duration for the SLE-2 group was $13.8 \pm 9.19$ years with a range of $2-33$ years. In terms of potential confounding influences on cognition, disease activity and corticosteroid doses were very low, hypertension was well controlled and distributed equally between groups, coronary artery disease was rare, no subjects had diabetes, and smoking occurred equally between groups. The mean prednisone dose was $2.5 \pm 3.4 \mathrm{mg}$, with a range of $0-10 \mathrm{mg}$. Based on body surface area, physiologic daily doses of prednisone for the SLE subjects would range from 2.1 to $4.8 \mathrm{mg}$ daily, and $84 \%$ of them were on a daily dose of less than or equal to $5 \mathrm{mg}$ daily. One subject in each group had anticardiolipin antibodies, and none had a lupus anticoagulant. Clinical data for the 13 SLE subjects included in the longitudinal assessments at baseline (time 1 [T1]) and time 2 (T2) are presented in Table 2. There were no significant changes in disease activity (SLEDAI scores), damage (SLICC DI scores), or medication use at the times the assessments were performed. Between T1 and T2, 5 of the 13 SLE subjects experienced a SLE flare requiring medication changes; 2 switched to different disease-modifying drugs; and 3 added disease-modifying drugs.

Cognitive and behavioral testing of SLE groups 1 and 2

There were no differences in mean throughput scores for the Automated Neuropsychological Assessment Metric (ANAM) subtests between the SLE groups (Table 1). However, although the SLE subjects had no 
Table 1. Subject characteristics for SLE-1 and SLE-2

\begin{tabular}{|c|c|c|c|}
\hline & SLE-1 $(n=17)$ & SLE-2 $(n=20)$ & $P$ value \\
\hline Age (yr) & $37.5 \pm 9.4$ & $41 \pm 10.3$ & 0.286 \\
\hline Sex: female & $17(100 \%)$ & $18(90 \%)$ & 0.489 \\
\hline \multicolumn{4}{|l|}{ Ethnicity/race } \\
\hline Latino/Hispanic & $7(41 \%)$ & $4(20 \%)$ & 0.160 \\
\hline Asian & $1(6 \%)$ & $1(5 \%)$ & 0.906 \\
\hline African American & $7(41 \%)$ & $14(70 \%)$ & 0.078 \\
\hline White & $2(12 \%)$ & $1(5 \%)$ & 0.564 \\
\hline Education (yr) & $13.5 \pm 4.3$ & $13.3 \pm 2.4$ & 0.839 \\
\hline SLEDAI score & $2.7 \pm 2.6$ & $2.3 \pm 2.1$ & 0.560 \\
\hline SLICC DI score & $0.8 \pm 0.8$ & $0.9 \pm 1.1$ & 0.675 \\
\hline \multicolumn{4}{|l|}{ Current medications } \\
\hline Prednisone dose (mg) & $2.4 \pm 3.1$ & $2.6 \pm 3.7$ & 0.821 \\
\hline Hydroxychloroquine & $12(71 \%)$ & $17(85 \%)$ & 0.428 \\
\hline DMARD $^{A}$ & $10(59 \%)$ & $11(55 \%)$ & 1.000 \\
\hline \multicolumn{4}{|l|}{ Serology } \\
\hline Anti-dsDNA $a b^{+}$ & $10(59 \%)$ & $10(50 \%)$ & 0.743 \\
\hline Anti-Ro ${ }^{+}$ & $7(30 \%)$ & $16(70 \%)$ & 0.021 \\
\hline Anti-NMDAR+ ${ }^{+}$ & $11(65 \%)$ & $9(45 \%)$ & 0.325 \\
\hline Anti-ribosomal $\mathrm{P}^{+}$ & $2(13 \%)$ & $3(15 \%)$ & 1.000 \\
\hline ACL (IgG, M, or A) & $1(6 \%)$ & $1(5 \%)$ & 0.459 \\
\hline \multicolumn{4}{|l|}{ Comorbid disease } \\
\hline Hypertension & $4(24 \%)$ & $9(45 \%)$ & 0.300 \\
\hline Coronaryartery disease & $1(6 \%)$ & $1(5 \%)$ & 1.000 \\
\hline Smoking: ever & $4(24 \%)$ & $7(35 \%)$ & 0.495 \\
\hline BMI & $26.5 \pm 5.0$ & $28.7 \pm 5.3$ & 0.195 \\
\hline \multicolumn{4}{|l|}{ Mood assessments } \\
\hline STAI ${ }^{B}$ & $35.8 \pm 9.9$ & $31.2 \pm 9.1$ & 0.151 \\
\hline BDI & $8.9 \pm 8.5$ & $7.4 \pm 5.4$ & 0.495 \\
\hline \multicolumn{4}{|l|}{ ANAM testing } \\
\hline Match-to-Sample & $21.4 \pm 11.2$ & $20.8 \pm 7.2$ & 0.834 \\
\hline Matching Grids & $27.9 \pm 10$ & $27.1 \pm 8.0$ & 0.783 \\
\hline $\mathrm{CPT}^{\mathrm{C}}$ & $74.2 \pm 36.3$ & $70.3 \pm 17.1$ & 0.685 \\
\hline \multicolumn{4}{|c|}{$\begin{array}{l}\text { ADisease-modifying anti-rheumatic drug; for SLE-1, methotrexate }(n=2) \text {, azathioprine }(n=3) \text {, mycophenolate mofe } \\
(n=5) \text {; for SLE-2, methotrexate }(n=1) \text {, azathioprine }(n=3) \text {, mycophenolate mofetil }(n=7) \text {. }{ }^{B} \text { State Trait Anxiety } \\
\text { Inventory, evaluates current anxiety. CRunning memory continuous processing test. SLE-1, SLE group 1; SLE-2, SLE } \\
\text { group 2; BDI, Beck Depression Inventory; ANAM, Automated Neuropsychological Assessment Metric. }\end{array}$} \\
\hline
\end{tabular}

prior diagnosis of cognitive impairment, in comparison with healthy control normative data (ANAM GNS Battery: Administration Manual and ANAM4 GNS: User Manual, Vista LifeSciences, unpublished observations), the SLE subjects demonstrated significantly lower scores on all 3 ANAM subtests ( $P=0.000$ for the Match-to-Sample and Matching Grids test and $P=0.006$ for the Running Memory Continuous Performance Test [CPT]). The Match-to-Sample subtest assesses visuospatial perception and working memory. $89 \%$ of the SLE subjects ( $82 \%$ of SLE-1, $95 \%$ of SLE-2, $P=0.315$ ) scored lower than the normative mean for this subtest and $19 \%$ (23\% of SLE-1, $15 \%$ of SLE-2, $P=0.68$ ) scored less than 2 SDs below the normative mean. The Matching Grids test assesses spatial processing efficiency. 92\% of the SLE subjects (88\% of SLE-1, $95 \%$ of SLE-2, $P=0.584)$ scored lower than the normative mean for this subtest, and $41 \%(41 \%$ of SLE-1, $40 \%$ of SLE-2, $P=1.0$ ) scored less than 2 SDs below the normative mean. The CPT assesses vigilance and sustained attention. $70 \%$ of the SLE subjects (53\% of SLE-1, 85\% of SLE-2, $P=0.069)$ scored lower than then normative mean for this subtest, and 13\% (23\% of SLE-1, 5\% of SLE-2, $P=0.159)$ scored less than 2 SDs below the mean. Both SLE groups had similar mean scores for depression and anxiety (Table 1); however, these did not reach cut off points associated with clinically significant symptoms (18, 19). The Beck Depression Inventory (BDI) has a range of scores from 0 to 63 (18). In SLE-1, the range for BDI scores was 0 to $31 ; 13$ subjects $(76 \%$ ) had BDI scores indicative of no depression (BDI scores $0-13$ ); 
Table 2. Clinical characteristics and cognitive test results of the 13 subjects in SLE-2 with imaging at T1 and T2, a mean of 14.9 months apart (range 11.3-19.1 months)

\begin{tabular}{|c|c|c|c|}
\hline & $\mathrm{T} 1(n=13)$ & $\mathrm{T} 2(n=13)$ & $P$ value \\
\hline SLEDAI score & $1.9 \pm 1.7$ & $3.0 \pm 2.8$ & 0.116 \\
\hline SLICC DI score & $1.1 \pm 1.1$ & $1.4 \pm 1.6$ & 0.104 \\
\hline \multicolumn{4}{|l|}{ Current medications } \\
\hline Prednisone dose (mg) & $2.9 \pm 4.1$ & $3.2 \pm 3.8$ & 0.828 \\
\hline Hydroxychloroquine & $10(77 \%)$ & $10(77 \%)$ & 1.000 \\
\hline DMARD $^{A}$ & $8(62 \%)$ & $11(85 \%)$ & 0.250 \\
\hline \multicolumn{4}{|l|}{ Mood assessments } \\
\hline STAI ${ }^{\mathrm{B}}$ & $30.5 \pm 9.6$ & $33.8 \pm 12.7$ & 0.437 \\
\hline $\mathrm{BDI}$ & $6.0 \pm 4.5$ & $7.5 \pm 5.8$ & 0.291 \\
\hline \multicolumn{4}{|l|}{ ANAM testing } \\
\hline Match-to-Sample & $19.9 \pm 7.1$ & $23.4 \pm 8.3$ & 0.016 \\
\hline Matching Grids & $27.0 \pm 8.9$ & $32.4 \pm 11.2$ & 0.036 \\
\hline $\mathrm{CPT}^{\mathrm{C}}$ & $71.2 \pm 20.2$ & $74.6 \pm 20.1$ & 0.496 \\
\hline
\end{tabular}

${ }^{A}$ Disease-modifying anti-rheumatic drug; for SLE-1, methotrexate $(n=2)$, azathioprine $(n=3)$, mycophenolate mofetil $(n=5)$; for SLE-2, methotrexate $(n=1)$, azathioprine $(n=3)$, mycophenolate mofetil $(n=7)$. ${ }^{B}$ State Trait Anxiety Inventory, evaluates current anxiety. ${ }^{C}$ Running memory continuous processing test. T1, time 1; T2, time 2; BDI, Beck Depression Inventory; ANAM, Automated Neuropsychological Assessment Metric.

$2(12 \%)$ had scores indicative of mild depression (BDI scores $14-19) ; 1(6 \%)$ had a score indicative of moderate depression (BDI scores 20-28); and $1(6 \%)$ had a score indicative of severe depression (BDI $\geq 29$ ). Similarly, in the SLE-2 group, the range of BDI scores was 0 to $16 ; 15$ (75\%) had scores indicative of no depression; and 5 (25\%) had scores indicative of mild depression. The range of scores for the State Trait Anxiety Inventory (STAI) measure is $20-80$ and a score $\geq 40$ indicates increased anxiety (19). In the SLE1 group, the range of scores was 20 to $52 ; 11(65 \%)$ of subjects had scores indicative of no anxiety; and 6 (35\%) had higher scores. For SLE-2, the range of scores was 20 to 50; 15 (75\%) had scores of less than 40; and $5(25 \%)$ had scores indicative of increased anxiety. The differences between groups for anxiety $(P=$ $0.719)$ and depression $(P=0.361)$ were not significant.

No significant differences were observed between $\mathrm{T} 1$ and $\mathrm{T} 2$ for the behavioral testing in the longitudinal studies (Table 2). Performance on two of the ANAM subtests, Match-to-Sample and Matching Grids, improved between $\mathrm{T} 1$ and $\mathrm{T} 2$ but still remained significantly below the normative mean scores.

Hypermetabolic regions identified in the combined SLE-1 and SLE-2 groups

Use of identical study methods allowed us to combine the FDG-PET data from SLE-1 and SLE-2 groups in a whole-brain, voxel-wise approach to compare the combined 37 SLE (SLE-1/2) and 25 healthy control (HC-1/2) subjects. SLE subjects demonstrated resting hypermetabolism (Figure 1 and Table 3 ) in the hippocampus (Figure 1, A and B; $P<0.001$ ), orbitofrontal cortex (BA 11) (Figure 1, C and D; $P<0.002$ ), and putamen/globus pallidus/thalamus (putamen/GP/thalamus) (Figure 1E; $P<0.001$ ), the same regions originally identified in the SLE-1 group (16) and independently validated in the SLE-2 group (Supplemental Data and Supplemental Table 1; supplemental material available online with this article; https://doi.org/10.1172/jci.insight.124002DS1). No significant differences were found between SLE-1 and SLE-2 subjects in these regions $(P>0.41)$. Identification of resting hypermetabolism in these same regions in SLE from the combined groups validates our previous results and, importantly, demonstrates the reproducibility and stability of the methodology.

Analysis of the combined groups, SLE-1/2 and HC-1/2, also identified 3 new hypermetabolic regions (Table 3): the sensorimotor cortex (SMC) (Figure 1F; $P<0.001$ ), occipital lobe (BA 19) (Figure 1G; $P$ $<0.002$ ), and temporal lobe (BA 37) (Figure 1H; $P<0.001$ ). No significant metabolic differences were present between SLE-1 and SLE-2 subjects in these hypermetabolic regions $(P>0.10)$. Moreover, hypermetabolism in the hippocampus and orbitofrontal cortex was highly abnormal bilaterally $(P<0.001)$. Hypermetabolism in the other 4 regions, though unilaterally abnormal at $P<0.001$, was also abnormal in the contralateral hemisphere at a lower significance level $(P<0.005)$.Longitudinal analysis of a subgroup of 13 SLE-2 subjects with baseline and follow-up FDG-PET scans demonstrated unchanged resting hypermetabolism $(P>0.21)$ in all 6 regions over a mean of 14.9 months. 

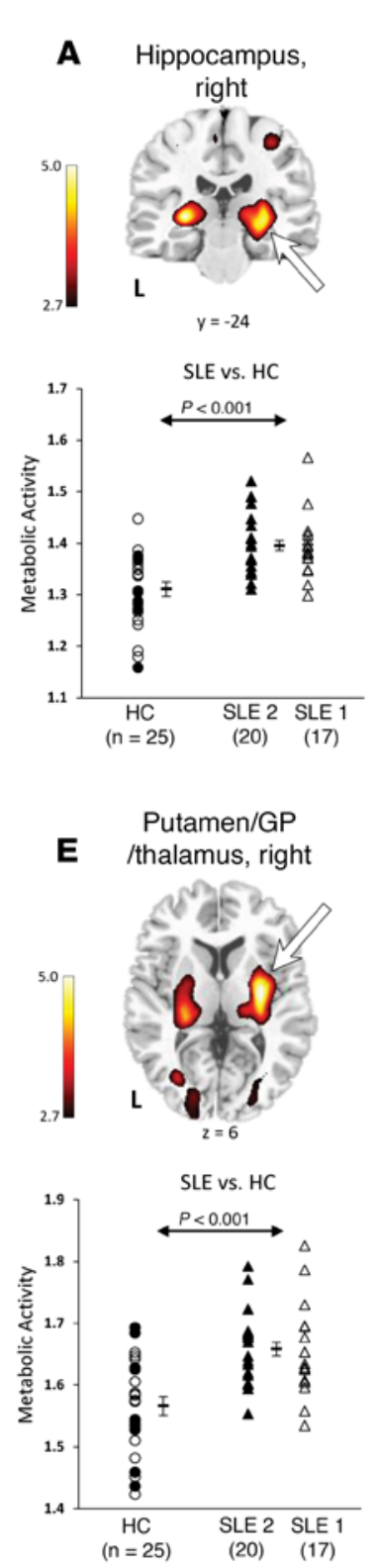
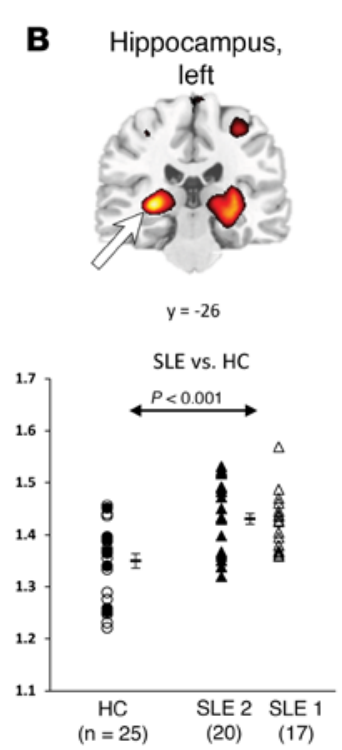

F $\begin{gathered}\text { Sensorimotor } \\ \text { cortex, right }\end{gathered}$
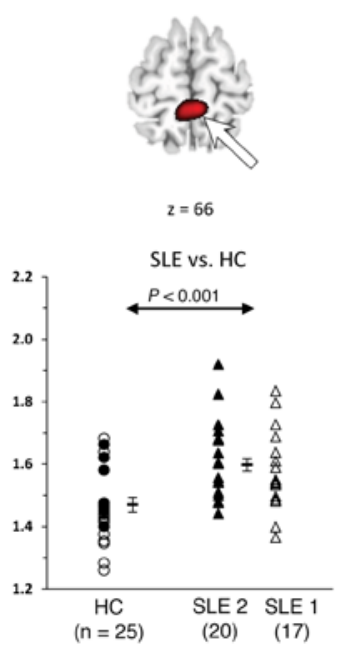
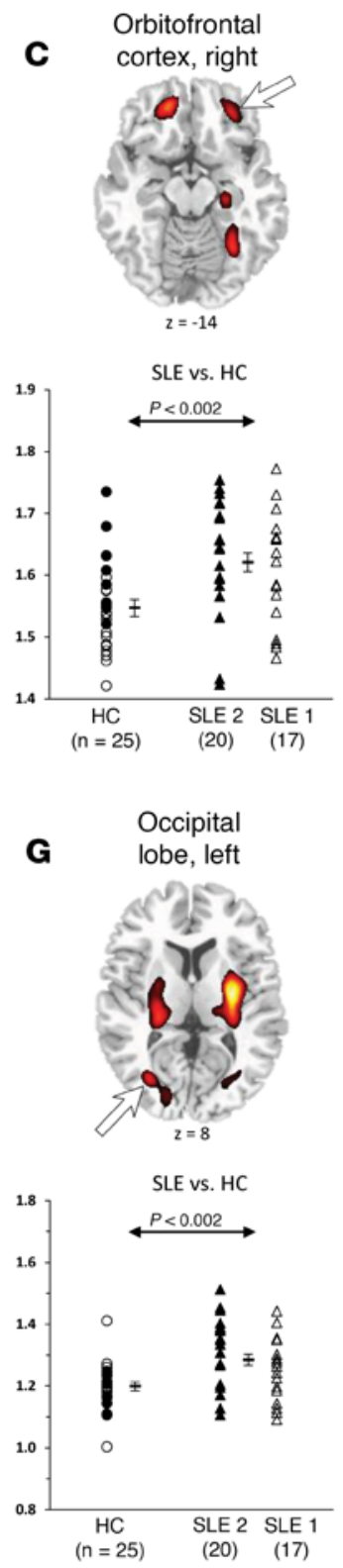
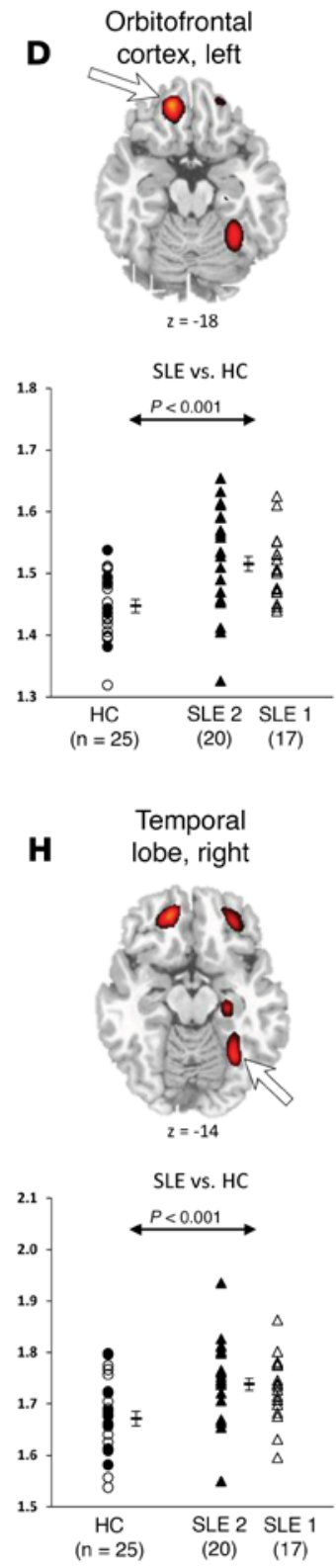

Figure 1. Abnormal hypermetabolic regions in SLE. Top: Voxel-wise comparison of the FDC-PET scans between the combined SLE-1/2 cohorts ( $n=37$ ) and healthy controls ( $\mathrm{HCs} ; n=25)$ revealed significant increases in resting glucose metabolism in SLE subjects in the hippocampus (A and B), orbitofrontal cortex (BA 11) (C and D), and putamen/GP/thalamus (E), the same regions independently identified in SLE-1 (1) and SLE-2 (Supplemental Data). Three new hypermetabolic regions identified in the SLE-1/2 subjects include the SMC (F), occipital lobe (BA 19) (G), and temporal lobe (BA 37) (H). (Peak voxel of each cluster was significant at $P<0.001$, uncorrected. Clusters for the hippocampus, putamen/GP/thalamus, and SMC were also significant at $P<0.05$, corrected for cluster extent [Table 3]. Clusters were displayed using a red-yellow scale thresholded at $P<0.005$ superimposed on a MRI template.). Bottom: Metabolism in these regions was significantly higher $(P<0.002)$ in the SLE-1/2 subjects (triangles) than in the healthy controls (circles) but not different between the SLE-1 (white triangles) and SLE-2 (black triangles) subjects $(P>0.41)$. (Error bar represents standard error of the mean. Arrow represents Student's $t$ test of SLE-1/2 subjects vs. HCs.)

\section{Altered interregional metabolic relationships in SLE and functional associations}

Interregional metabolic correlations were examined among the 6 hypermetabolic regions identified from the SLE- $1 / 2$ and HC- $1 / 2$ analyses. HC-1/2 subjects demonstrated significant interregional metabolic correlations between the hippocampus and putamen/GP/thalamus $(\mathrm{r}=0.60, P<0.002)$, the hippocampus and temporal lobe $(\mathrm{r}=0.40, P<0.05)$, and the putamen/GP/thalamus and temporal lobe $(\mathrm{r}=0.49, P$ $<0.02$ ) (Figure 2A). SLE-1/2 subjects also demonstrate a significant metabolic correlation between the hippocampus and putamen/GP/Thalamus $(r=0.45, P<0.006)$. However, other interregional metabolic 
Table 3. Brain regions with significant differences in metabolic activity between 37 SLE subjects from the combined groups 1 and 2 (SLE-1/2) and 25 healthy controls (HC-1/2)

\begin{tabular}{|c|c|c|c|c|c|c|c|}
\hline \multicolumn{8}{|c|}{ Coordinates $^{A}$} \\
\hline Brain region & & $x$ & y & z & $Z_{\max }^{B}$ & $\mathrm{HC}(n=25)$ & $\operatorname{SLE}(n=37)$ \\
\hline \multirow[t]{2}{*}{ Hippocampus } & Right & 28 & -20 & -2 & $4.69^{c}$ & $1.31(0.07)^{\mathrm{D}}$ & $1.40(0.06)^{\mathrm{F}}$ \\
\hline & Left & -28 & -24 & 0 & $4.56^{c}$ & $1.35(0.07)$ & $1.43(0.06)^{\mathrm{F}}$ \\
\hline \multirow[t]{2}{*}{ Orbitofrontal cortex (BA 11) } & Right & 30 & 40 & -14 & 3.50 & $1.55(0.07)$ & $1.62(0.09)^{\mathrm{E}}$ \\
\hline & Left & -16 & 44 & -18 & 3.88 & $1.45(0.05)$ & $1.52(0.07)^{F}$ \\
\hline Putamen/GP/thalamus & Right & 30 & -12 & 4 & $4.68^{c}$ & $1.57(0.08)$ & $1.66(0.07)^{\mathrm{F}}$ \\
\hline Temporal lobe (BA 37) & Right & 30 & -58 & -14 & 3.56 & $1.67(0.07)$ & $1.74(0.07)^{F}$ \\
\hline
\end{tabular}

correlations in the SLE-1/2 subjects shift away from the temporal lobe, as seen in the HCs, to include significant correlations between the SMC and hippocampus ( $\mathrm{r}=0.38, P<0.03)$ and putamen/GP/thalamus ( $\mathrm{r}$ $=0.34, P<0.04)$ (Figure $2 \mathrm{~B}$ ). There were no significant interregional correlations between the orbitofrontal cortex (HC: $P>0.07$; SLE: $P>0.19$ ) and the occipital lobe (HC: $P>0.13$; SLE: $P>0.14$ ) and any other hypermetabolic regions identified in SLE-1/2. These results suggest that a normal resting pattern of interregional metabolic relationships is altered among abnormal hypermetabolic regions in SLE.

Correlations between metabolic activity in the 6 hypermetabolic regions and clinical and behavioral measures in the SLE-1/2 subjects were also examined. Poor performance on the running memory CPT, a measure of working memory and attention, correlated with increased resting metabolism in the hippocampus ( $\mathrm{r}=-0.35, P<0.04)$, validating the previously reported relationship between these two measures (16). We also found new inverse correlations of poor performance on the CPT with increased metabolism in other hypermetabolic areas, including the SMC $(\mathrm{r}=-0.42, P<0.02)$, putamen $/ \mathrm{GP} /$ thalamus $(\mathrm{r}=-0.32, P$ $=0.05)$, temporal lobe $(\mathrm{r}=-0.35, P<0.04)$, and occipital lobe $(\mathrm{r}=-0.39, P<0.02)$. Depression $(\mathrm{r}=0.36$, $P<0.03)$ and anger $(\mathrm{r}=0.34, P<0.04)$ on the ANAM mood scale correlated with hypermetabolism in the orbitofrontal cortex. Additionally, the SLICC Damage Index correlated with abnormal hypermetabolism in the hippocampus $(\mathrm{r}=0.37, P<0.03)$. Mean scores on the CPT test did not change significantly between $\mathrm{T} 1(71.2 \pm 20.2)$ and T2 $(74.6 \pm 20.1)(P=0.496)$ in the 13 SLE-2 subjects with repeat scans (Table 2$)$.

\section{Correlations of regional metabolism with disease duration}

In our prior study of SLE-1 subjects (16), decreased metabolism in the prefrontal (BA 9 and 10) and premotor (BA6) cortex correlated significantly with longer disease duration. In the SLE-1/2 subjects, similar inverse correlations with disease duration and metabolism were found in the prefrontal cortex (BA 9: $\mathrm{r}=-0.35, P<$ 0.04; BA 10: $\mathrm{r}=-0.30, P=0.07$ ) and premotor cortex (BA $6, \mathrm{r}=-0.66, P<0.0001$ ). Among the 6 hypermetabolic regions in SLE-1/2, disease duration correlated only modestly with hypermetabolism in the SMC $(\mathrm{r}=$ $0.33, P<0.05)$. These results suggest that decreasing metabolism in the prefrontal and premotor cortices may be a function of disease chronicity and damage rather than active immune-related processes.

\section{DTI results}

Microstructural changes - cross-sectional and longitudinal. Significant reductions in fractional anisotropy (FA) in the SLE-2 group, relative to 14 age- and sex-matched HCs $(\mathrm{HC}-3)(P<0.001)$, identified 5 discrete regions, or clusters, with abnormal WM microstructure in the SLE-2 subjects (Figure 3 and Table 4). The clusters in the parietal lobe, the cingulum (hippocampus), and the splenium corpus callosum (CC) were significant at $P<0.001$, corrected for cluster extent at $P<0.05$. Cluster 1 was localized to the WM tracts in the parietal lobe, which is a part of the superior longitudinal fasciculus (SLF) connecting the frontal, occipital, parietal, and temporal lobes. Cluster 2 was localized to the WM tracts in the vicinity of the insular cortex, which is a part of the uncinate fasciculus (UF), connecting parts of the limbic system, such as 
A

HC- $1 / 2$

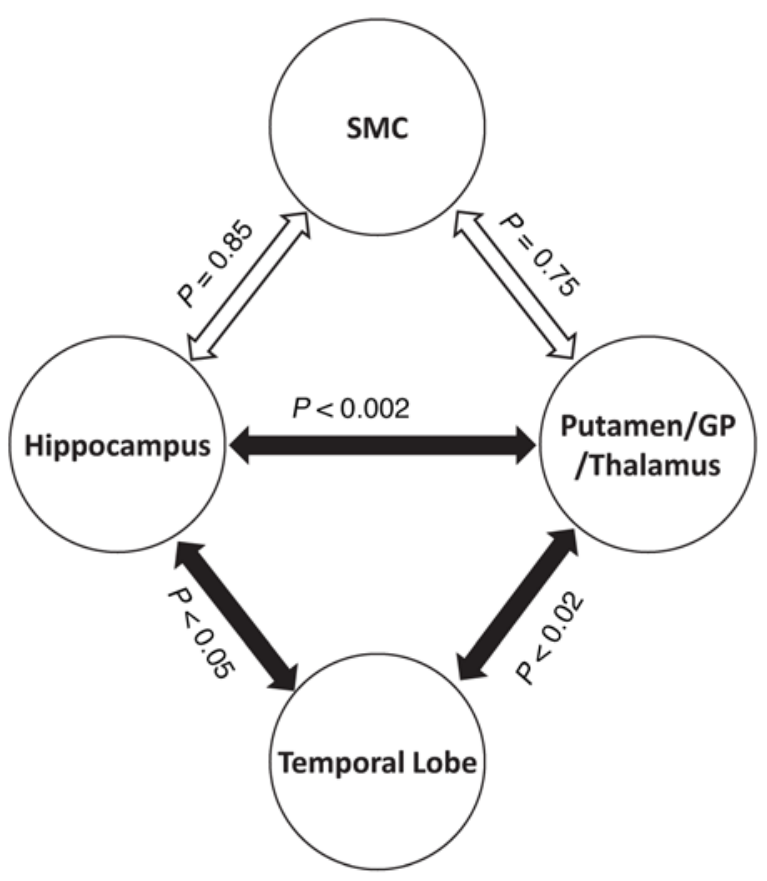

B

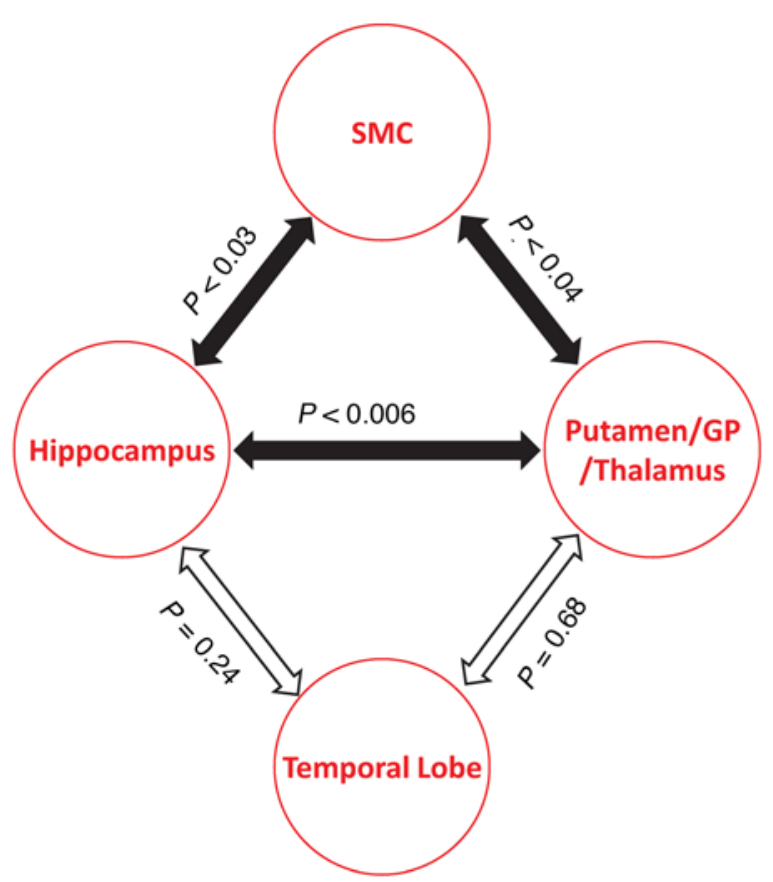

Figure 2. Altered pattern of interregional metabolic correlations between abnormal hypermetabolic regions in SLE. (A) Healthy controls (HC) demonstrate a normal pattern of interregional metabolic correlations such that resting metabolism among the hippocampus, putamen/GP/thalamus, and temporal lobe are all significantly correlated (filled arrows; $P<0.05$ ). (B) In contrast, while the significant hippocampal-putamen/GP/thalamic metabolic relationship $(P<0.006)$ is preserved in SLE, other significant metabolic correlations shift away from the temporal lobe to include the SMC $(P<0.04)$.

the hippocampus and amygdala in the temporal lobe, with frontal lobe elements, such as the orbitofrontal cortex. Cluster 3 was localized to the WM tracts in the occipital lobe, which is a part of the cingulum that is connected to the hippocampus and a part of the inferior longitudinal fasciculus (ILF), which is connected to the temporal lobe and occipital lobe. Cluster 4 was in the WM tracts in the frontal lobe, which is a part of the inferior frontal occipital fasciculus (IFOF). The last abnormal region, cluster 5, was localized in the WM tracts in the parietal lobe, which is a part of the splenium of CC. Of note, the FA reductions observed in these regions were also significant $(P<0.05)$ in homologous areas in the contralateral brain.

To evaluate the spatial relationships of clusters with significantly abnormal WM microstructure (low FA) in the SLE-2 group with corresponding hypermetabolic gray matter regions in these patients, we overlaid statistical parametric maps of the SLE-2 and HC-3 group contrasts, thresholded at T $=3.0(P<0.005)$ for metabolic activity (SLE $>$ HC) and FA (SLE $<$ HC) on a common MRI template (Figure 4). We found that 4 of the clusters with significant SLE-related FA reductions (the SLF parietal cluster, the UF insular cluster, the ILF occipital cluster, the IFOF frontal cluster, see Figure 3 and Table 4) lay in close proximity to areas of SLE-related hypermetabolism in the adjacent gray matter (Figure 4, yellow ellipses).

There were no significant changes in microstructural integrity FA values over a mean of 14.9 months in the subgroup of $13 \mathrm{SLE}$ subjects with follow-up scans $(P>0.3$, paired $t$ tests) in all $5 \mathrm{WM}$ regions (Table 4$)$.

Localization of tract changes. Each of the abnormal FA clusters was used as a seed region for fiber tracking. In each pathway, fewer tracts were visualized in the SLE-2 compared with HC-3 subjects (Figure 5). SLE-2 subjects demonstrated reduced tract numbers in the SLF (temporal part) $(-74 \%)$, the UF $(-86 \%)$, the cingulum (hippocampus part) $(-82 \%)$, the ILF $(-99.5 \%)$, IFOF $(-100 \%)$, and the splenium CC $(-48 \%)$.

Correlations between microstructural integrity and serum DNRAb titers and spatial memory performance. As expected, performance of SLE-2 subjects on the $2 \times 2$ spatial memory task ( $2 \times 2$ SMT) $(74 \% \% \pm 13 \%$ correct) was significantly worse than on the $2 \times 2$ nonspatial memory task $(93 \% \pm 11 \%$ correct; $P=0.014)$. We examined relationships among serum DNRAb titers, $2 \times 2$ SMT performance, and microstructural integrity. Serum DNRAb titer was entered as a covariate in a voxel-wise search for regions in which FA correlated with serum DNRAb 

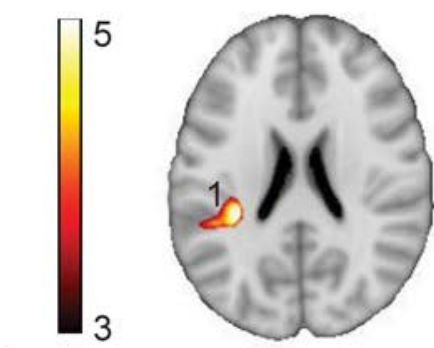

t-values
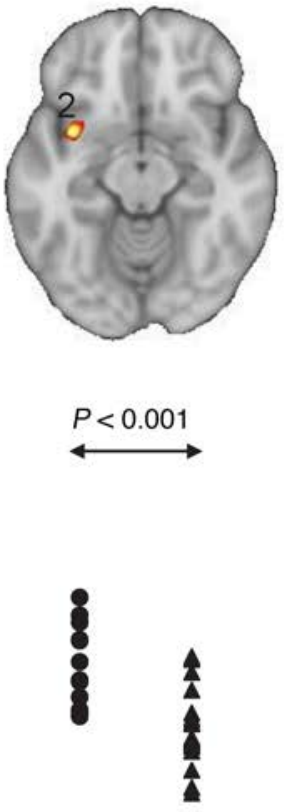

HC SLE
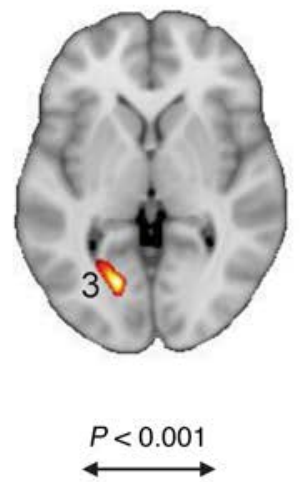

슬
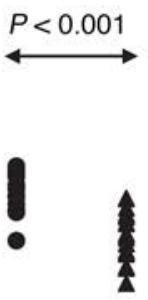

HC SLE
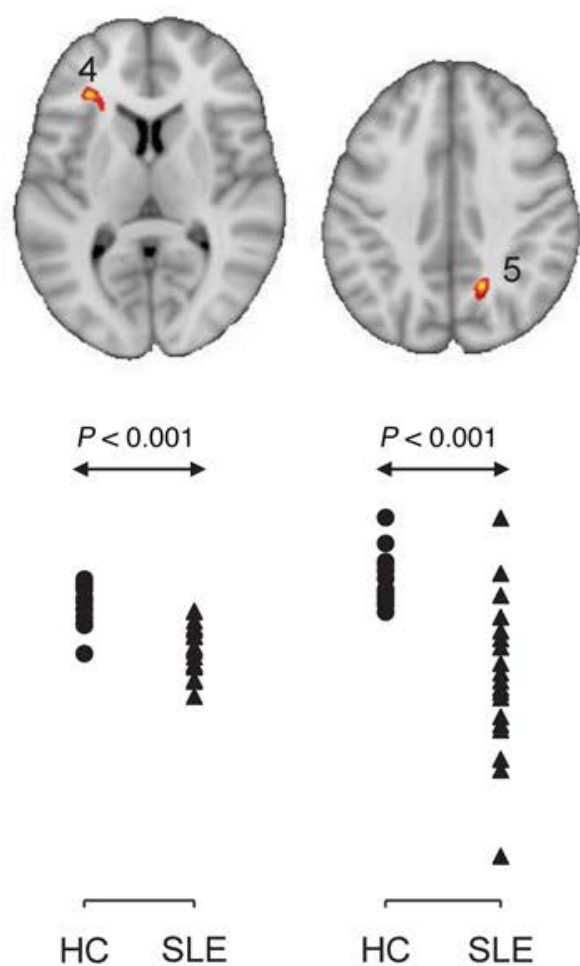

Figure 3. Regions with abnormal microstructure in SLE subjects. Significant reductions $(P<0.001)$ in FA were present in the following brain areas (top): 1 , white matter tracts in the parietal lobe, a part of the superior longitudinal fasciculus (SLF); 2 , white matter tracts in the vicinity of the insular, a part of the uncinate fasciculus (UF); 3 , white matter tracts in the occipital lobe/cingulum (hippocampus); 4, white matter tracts in the frontal lobe, a part of the inferior frontal occipital fasciculus (IFOF); and 5, white matter tracts in the parietal lobe, a part of the splenium of corpus callosum (CC). Individual cluster fractional anisotropy (FA) values for the SLE and control groups were plotted (bottom). All group differences were highly significant $(P<0.001)$. The details of these brain regions were presented in Table 4.

titer. A significant correlation $(P<0.002)$ was identified in the bilateral parahippocampal area (BA 36/BA 20, Figure 6A, yellow/red). Mean FA in these areas exhibited a negative correlation $(\mathrm{r}=-0.64, P<0.002)$ with serum DNRAb titers (Figure $6 \mathrm{~B})$ and a positive correlation $(\mathrm{r}=0.63, P<0.002)$ with $2 \times 2$ SMTscores (Figure $6 \mathrm{~B})$. Moreover, a significant inverse correlation $(\mathrm{r}=-0.46, P<0.05)$ was found between hypermetabolism in the hippocampus, identified in the FDG-PET studies of the same SLE-2 subjects (Supplemental Table 1), and $2 \times 2$ SMT scores. In aggregate, these data suggest that poor performance on the spatial memory task is significantly associated with hippocampal hypermetabolism and decreased microstructural integrity in the parahippocampal region that is also associated with high serum DNRAb titers.

\section{Discussion}

We have employed an integrated approach, using resting-state functional and structural neuroimaging techniques and neuropsychological testing, to characterize CD in lupus patients with quiescent, stable disease and no known history of acute NPSLE or CNS dysfunction. Despite the absence of a known diagnosis of NPSLE syndromes, the majority of SLE subjects enrolled in this study scored below the mean of healthy control normative data on the ANAM subtests, consistent with prior reports of the high prevalence of CD in SLE (20). This is an important distinction, given the associations reported here among cognitive performance, abnormal brain resting metabolism, and structure. We have validated our initial findings of abnormal resting hypermetabolism in the hippocampus, orbitofrontal cortex, and putamen/GP/thalamus of SLE subjects as well as in 3 new regions: the SMC, occipital lobe, and temporal lobe. The resting pattern of interregional metabolic relationships among these hypermetabolic areas is abnormally skewed in SLE compared with HC subjects, and hypermetabolism in 5 of the regions correlates significantly with impaired performance on a test of working memory. Consistent with the metabolic data, FA analysis reveals 4 clusters of decreased microstructural integrity localized in WM tracts adjacent to the hypermetabolic regions, 
Table 4. Brain regions with significant differences in FA between 14 healthy controls (HC-3) and 19 SLE-2 subjects at baseline (TP1) and stability of FA measures in 13 SLE-2 subjects over a mean of 15 months from TP1 to TP2

\begin{tabular}{|c|c|c|c|c|c|c|c|}
\hline \multicolumn{8}{|c|}{ Coordinates $^{A}$} \\
\hline Brain region & $x$ & y & z & $Z_{\max }^{B}$ & $\mathrm{HC}(n=14)$ & $\begin{array}{l}\text { SLE TP1 }(n \\
\quad=19)\end{array}$ & $\begin{array}{l}\text { SLE TP2 }(n \\
\quad=13)\end{array}$ \\
\hline Parietal lobe, left, SLF & -33 & -35 & 22 & $4.66^{\mathrm{C}}$ & $0.38(0.03)^{\mathrm{D}}$ & $0.32(0.03)$ & $0.32(0.03)$ \\
\hline Insular, left, UF & -36 & 5 & -13 & 4.33 & $0.28(0.03)$ & $0.23(0.02)$ & $0.22(0.03)$ \\
\hline $\begin{array}{l}\text { Cingulum (hippocampus), } \\
\text { left, ILF }\end{array}$ & -18 & -69 & -1 & $4.29^{\mathrm{C}}$ & $0.32(0.03)$ & $0.25(0.02)$ & $0.24(0.02)$ \\
\hline Frontal lobe, left, IFOF & -31 & 33 & 8 & 4.02 & $0.38(0.02)$ & $0.34(0.02)$ & $0.34(0.02)$ \\
\hline $\begin{array}{l}\text { Parietal lobe, right, } \\
\text { Splenium CC }\end{array}$ & 17 & -57 & 38 & $3.96^{c}$ & $0.40(0.02)$ & $0.32(0.06)$ & $0.32(0.06)$ \\
\hline \multicolumn{8}{|c|}{$\begin{array}{l}{ }^{A} \text { Montreal Neurological Institute standard space (60). }{ }^{B} \text { Significant at } P<0.001 \text { (peak voxel, uncorrected), cluster extent } \\
\text { test. 'Significant at } P<0.05 \text {, corrected for for cluster extent test. }{ }^{D} \text { Mean (SD) regional fractional anisotropy (FA). In all } \\
5 \text { brain regions, when comparing SLE- } 2 \text { with healthy control subjects, } P<0.001 \text {, Student's } t \text { test. FA was also assessed } \\
\text { in the same clusters in } 13 \text { of the SLE- } 2 \text { subjects a mean of } 15 \text { months later at TP2. When comparing FA in all } 5 \text { clusters } \\
\text { from these } 13 \text { SLE-2 subjects at TP1 to TP2, } P>0.3 \text {, paired Student's } t \text { test. HC, healthy controls; SLE, systemic lupus } \\
\text { erythematosus; TP, time point; SLF, superior longitudinal fasciculus; UF, uncinate fasciculus; ILF, inferior longitudinal } \\
\text { fasciculus; IFOF, inferior frontal occipital fasciculus; CC, corpus callosum. }\end{array}$} \\
\hline
\end{tabular}

and the connecting tracts are significantly diminished in the SLE subjects. Decreased microstructural integrity in the parahippocampal gyrus correlates with impaired spatial memory and increased serum DNRAb titers. In aggregate, these results suggest that CD in SLE is characterized by a diffuse process that results in reproducible resting hypermetabolism in several gray matter regions and disruption of normal resting metabolic patterns that associate with (a) poor performance on cognitive testing and (b) clusters of decreased microstructural integrity that are connected by diminished WM tracts. While many inflammatory molecules associated with SLE have the potential to damage neurons through direct neurotoxicity, microglial activation, or other mechanisms $(10,12,13)$, it is not surprising that antibodies targeting the NMDA receptor (DNRAb) are significantly associated with impaired spatial memory and abnormalities in glutamatergic brain regions, such as the hippocampus and parahippocampus.

There have been many studies utilizing FDG-PET imaging in SLE and a variety of NPSLE syndromes that, in contrast to our results, have reported regional hypometabolism in the frontal, temporal, parietal, and parietal-occipital regions (21-25). Regional gray matter hypermetabolism has been reported rarely in the striatal nuclei and basal ganglia, typically in the clinical context of SLE patients with choreoathetoid movements (23, 26, 27). Several important differences in methodology may account for the dissimilarities with our results. Each of these previous studies has imaged SLE subjects with active disease, with or without NPSLE (focal deficits, acute confusional state, mood disorders, $\mathrm{CD}$, seizures), with attendant treatment regimens, including high doses of glucocorticoids and immunosuppressive medications. The analyses done in these studies have been based on measures of glucose metabolism in prespecified regions of interest (ROIs), which were applied to each scan, and comparisons were either between active SLE and active NPSLE or healthy controls. Our analytic approach employed an unbiased, voxel-wise search of whole-brain volumes to directly compare SLE and healthy control subjects. This approach may have allowed for greater sensitivity and objectivity in detecting differences between groups than an approach that uses predetermined ROIs. Additionally, we studied SLE subjects with no history of active or prior CNS events, relatively few significant comorbid diseases, or high levels of antiphospholipid antibody titers, with low/stable disease activity, thereby avoiding confounding influences of medications, comorbid disease, and disease activity, whether neuropsychiatric or peripheral. The reproducibility of regional hypermetabolism in the hippocampus, orbitofrontal cortex, and putamen/GP/thalamus in both the separate and combined SLE cohorts validates the stability of our methodology and suggests that hypermetabolism represents a SLE-associated biologic process in these regions. Interestingly, several prior FDG-PET studies reported follow-up scans that demonstrated improvement or normalization of the previously found hypometabolic regions in subjects with clinical improvement (23-25). It is therefore likely that the regional hypometabolism was a function of acute illness, whether a specific NPSLE syndrome or not, as it is known that many inflammatory 


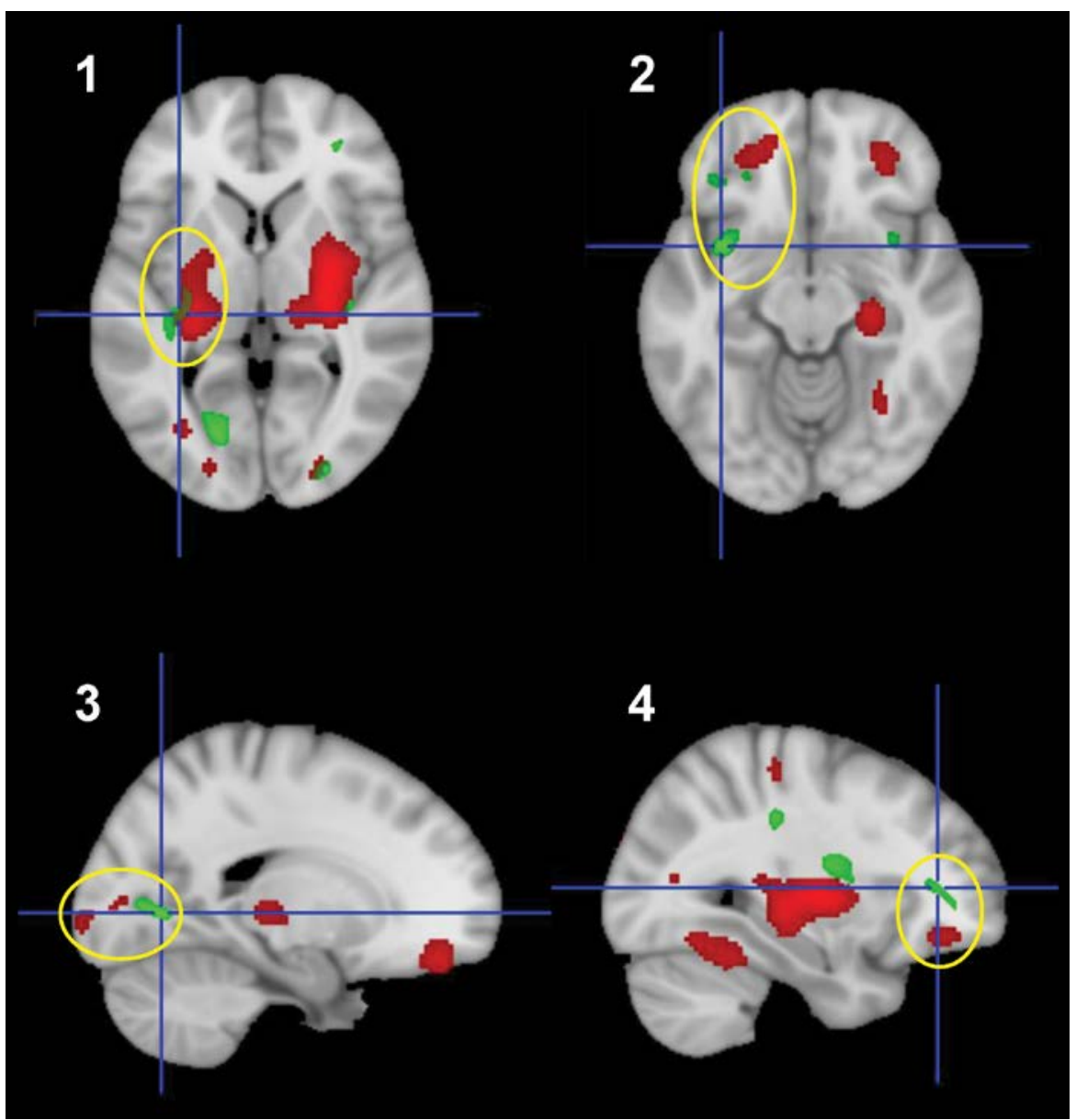

Figure 4. Spatial proximity of white matter regions with abnormal reductions in fractional anisotropy in SLE-2 patients and areas of increased metabolic activity in adjacent gray matter. White matter regions with abnormal reductions in fractional anisotropy are represented in green (clusters 1-4, see Figure 3 and Table 4); areas of increased metabolic activity in adjacent gray matter are represented in red (see Figure 1 and Table 3. Adjacent gray and white matter regions are encompassed by yellow ellipses. (Clusters represent areas with significant differences in local fractional anisotropy [FA] or metabolic activity in SLE vs. healthy control subjects [thresholded at $\mathrm{T}=3.0, P<0.005$ ], overlaid on the MNI152 T1 MRI template.).

molecules associated with disease flares can affect CNS function (reviewed in ref. 12). Follow-up scans in 13 of our SLE subjects, with low disease activity at the time of imaging, demonstrated no significant change over a mean of 14.9 months, despite evidence of increased interim SLE disease activity in 5 of these 13 subjects. These data suggest that the persistent hypermetabolism may be intrinsic to SLE and is not associated with disease duration or disease activity. This is in contrast to the hypometabolic regions in the prefrontal and premotor cortices, identified in all 3 SLE groups, that correlated with disease duration, suggesting an association between hypometabolism and chronic damage. We hypothesize that the stability of the regional metabolic overactivity appears to be a reproducible, stable marker for SLE-related CD that may be responsive to targeted therapies and therefore potentially useful as an outcome measure in a clinical trial.

Increased PET signal indicates metabolic overactivity that may represent regional neuronal activity, local inflammatory or oncogenic activity, or disinhibition after damage to remote but connected regions (28-31). Turning to preclinical experiments for guidance, it has been demonstrated in micro-FDG-PET studies of nonautoimmune mice immunized to produce serum DNRAb that affected mice have decreased metabolism in the hippocampus in the first 2 weeks after blood-brain barrier disruption, coincident with histological study of neuronal toxicity and death. This is followed over 9 weeks by steadily increasing hippocampal metabolism that correlates inversely with hippocampal CA1 neuron number, verified with unbiased quantitative histopathology. These findings suggest a delayed tissue response - either a com- 


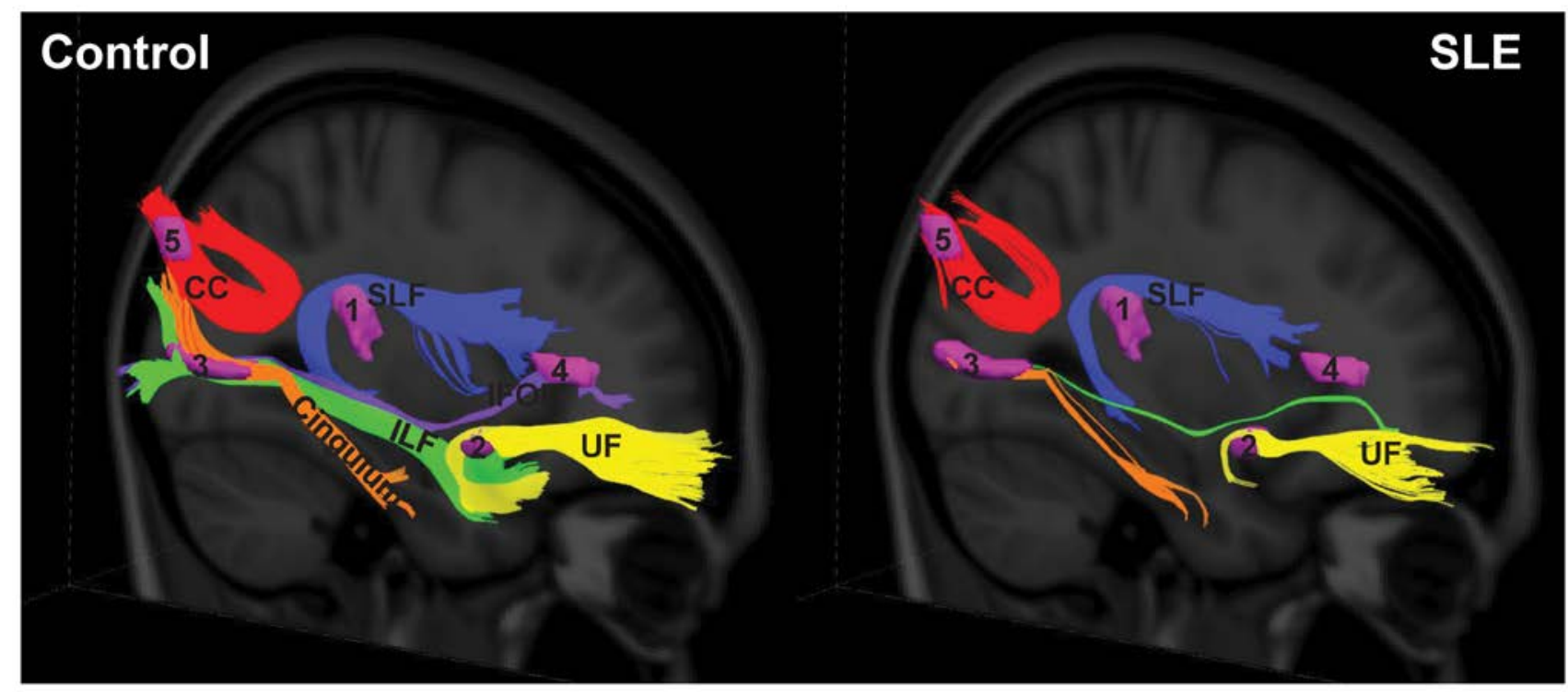

Figure 5. White matter pathways associated with the abnormal SLE-related regions visualized with group tractography. The superior longitudinal fasciculus (temporal part) (SLF) (noted as 1), uncinate fasciculus (UF) (noted as 2), cingulum (hippocampus part) and inferior longitudinal fasciculus (ILF) (noted as 3), inferior frontal occipital fasciculus (IFOF) (noted as 4), and the splenium of the corpus callosum (CC) (noted as 5) pathways reconstructed in the healthy control (left) and SLE (right) groups. Fewer tracts were visualized in the SLE group relative to the controls in the SLF (temporal part) (-74\%), UF (-86\%), cingulum (hippocampus part) (-82\%), ILF (-99.5\%), IFOF (-100\%), and splenium CC (-48\%).

pensatory neuronal mechanism or an inflammatory mechanism such as recruitment of microglial cells to sites of neuronal necrosis (32). These preclinical data provide a cautionary tale in the interpretation of cross-sectional human data. We have identified several abnormal hypermetabolic regions in SLE that do not associate with age, disease duration, current medication use, or disease activity; that exhibit abnormal metabolic relationships between each other; and associate with poor performance on a memory task, but we cannot know whether the hypermetabolism is representative of a compensatory neuronal response, decreased inhibitory input from another damaged region, or a microglial response to damaged neurons or other inflammatory molecules.

This is relevant to the shift in interregional metabolic relationships away from the temporal lobe to include the SMC that we report in SLE subjects. Possible explanations for this shift include damage to the temporal lobe, damage to the SMC with influx of microglial cells, and compensatory neuronal response or damage elsewhere resulting in decreased inhibitory input to the SMC. Nonetheless, these data suggest, perhaps, an erosion of normal, resting-state brain activity that characterizes SLE patients and associates with impaired performance on cognitive testing. Once a significant resting pattern has been identified, particularly one that is not associated with disease duration, disease activity, medications, or comorbid disease, it may be used for early disease detection, to discriminate between groups, to evaluate response to treatment strategies, or to assess disease progression.

Integration of the PET metabolic data and the DTI structural data suggests correlations between regional hypermetabolism and areas of diminished WM microstructural integrity that may account for the shift in interregional metabolic relationships. DTI analyses identified 4 WM clusters: the parietal lobe involving the SLF, the insula involving the UF, the occipital lobe involving the ILF, and the frontal lobe involving the IFOF. These areas of abnormally decreased FA in WM were in close proximity to hypermetabolic regions in the adjacent gray matter (Figure 4, ellipses). For each of these projection pathways, tractography (see Methods) revealed fewer tracts in the SLE-2 sample relative to HC-3 control subjects. Our interpretation of these compelling data is that the original insult may take place in the hypermetabolic, gray matter regions, followed by disruption of the WM tracts connecting them. Additional longitudinal studies are needed to verify this prediction. While several studies have reported WM alterations in NPSLE compared with HC subjects in segments of the CC, the forceps, anterior corona radiata, thalamus, and parietal and frontal lobes (33-36) and overall diminished volume of the CC $(34,37)$, our results support the 
A

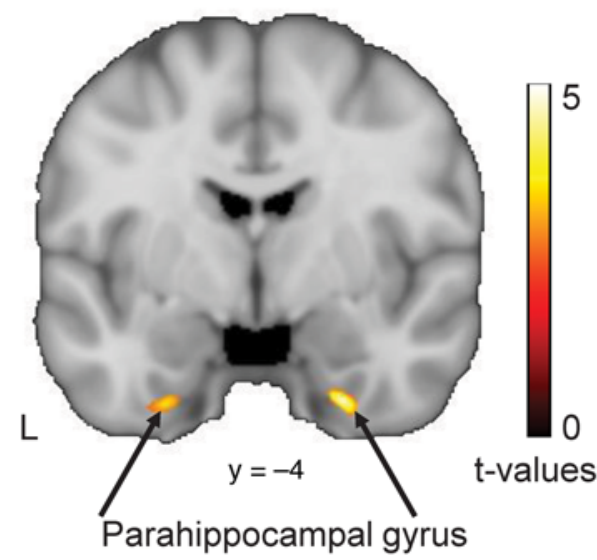

B
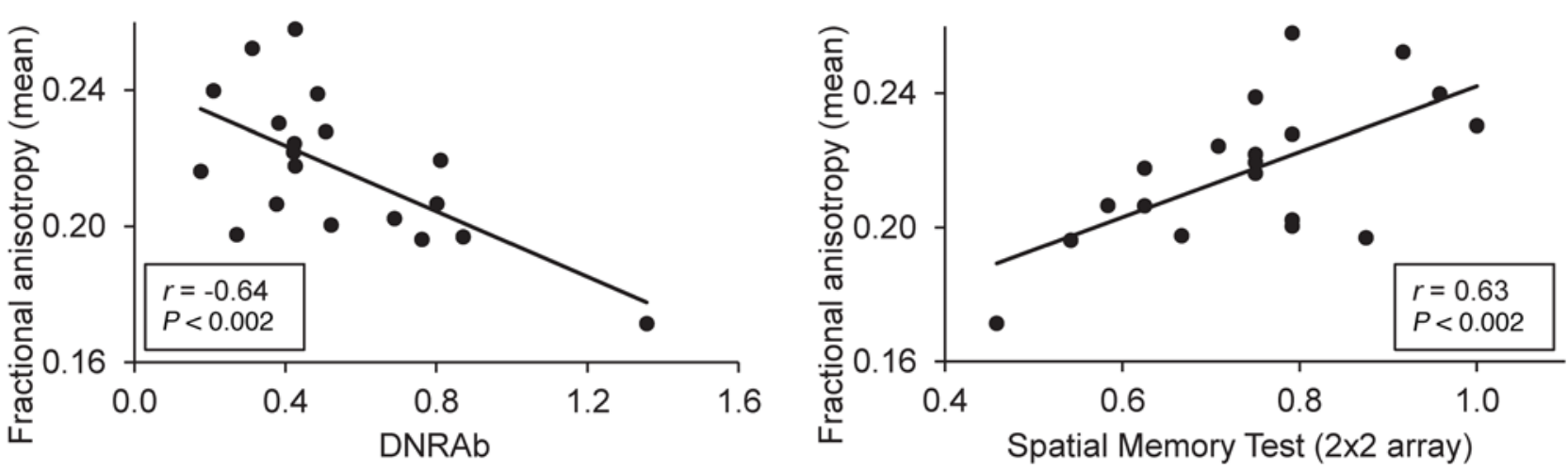

Figure 6. Microstructural integrity (FA) in the parahippocampus regions correlates with serum DNRAb titers and performance on a spatial memory test in SLE subjects. (A) Regression analysis of voxel-wise correlations between FA maps and serum DNRAb titers revealed a significant inverse correlation between FA values in the bilateral parahippocampal gyrus (BA 36/BA 20; yellow/red areas) and serum DNRAb titers. (B) Mean microstructural integrity in the bilateral parahippocampal gyrus exhibited a negative correlation with serum DNRAb titers $(r=-0.64, P<0.002$, left). Mean microstructural integrity in the bilateral parahippocampal gyrus also exhibited a positive correlation with performance on a spatial memory test $(r=063, P<0.002$, right). Pearson's product-moment correlation coefficient was used to evaluate the correlations between FA values and serum DNRAb titers and 2x2SMT scores.

growing body of literature demonstrating significant WM abnormalities in SLE subjects without acute neuropsychiatric manifestations (38-42). Most of these studies in SLE subjects without neuropsychiatric manifestations failed to demonstrate significant correlations between diminished WM integrity and cognition. However, Shapira-Lichter et al. have reported impaired performance on a learning and verbal memory task that correlated with decreased integrity of WM tracts in the CC and cingulum in SLE subjects without neuropsychiatric symptoms (40). We have identified a significant correlation between reduced FA in the parahippocampal gyrus, poor performance on a spatial memory task, and elevated serum DNRAb titers. Poor performance on the 2x2SPMT also correlated with hippocampal hypermetabolism. These results suggest the importance of selective cognitive testing based on biologic mechanisms rather than predetermined cognitive batteries that may not capture the specific cognitive deficit related to a particular mechanism. The hippocampus, the center for memory processing and storage (43), is one of the regions with reproducible hypermetabolic results that we have identified in SLE subjects. Hippocampal abnormalities have been well described in SLE patients and animal models. In human SLE, hippocampal atrophy, presumably reflective of neuronal loss, has been correlated with cognitive deficits $(38,44-48)$ as well as specific autoantibodies, including DNRAb $(44,49)$. Our findings of reproducible hippocampal hypermetabolism with functional correlates extend these previous reports, suggesting an SLE-mediated attack on the hippocampus. In animal models, neuronal damage and atrophy in the hippocampus in lupus mice correlates with impaired spatial learning (50) that is prevented with immunosuppressive therapy (51). Selective targeting of hippocam- 
pal neurons by DNRAb in the mouse model, specifically place cells in the CA1 region of the hippocampus, results in cognitive and spatial memory deficits $(15,52)$. We previously reported a significant association in SLE patients between serum DNRAb titers and impaired performance on the 2x2SMT (15). We currently report a significant correlation between performance on the 2x2SMT and hippocampus metabolism. In addition, identification of correlations between reduced microstructural integrity in the parahippocampal gyrus and poor performance on the 2x2SMT as well as high serum DNRAb titers in SLE subjects further supports the hypothesis that antibody-mediated neuronal toxicity may induce damage to the hippocampal region that results in disorganization and reduced integrity of the WM outflow tract through the parahippocampus and manifests functionally as impaired spatial memory.

Our study is limited by the use of historical healthy controls; although they are age- and sex-matched and their PET/MRI imaging protocols were identical to those used for the SLE subjects, we do not have cognitive testing on these individuals or information regarding ethnicity, race, and socioeconomic status. Additionally, the lack of functional imaging during cognitive testing limits our interpretations to asynchronous correlations between task performance and integrity of the neuroanatomy. This was an intentional choice, as we are unable to ensure subject motivation during testing in a scanner; however, we acknowledge that functional imaging during testing would provide additional valuable information.

In summary, this is the first study to our knowledge to examine both gray and WM abnormalities together in SLE in concert with cognitive assessments. We have used structural and functional imaging to demonstrate the reproducibility of regional resting hypermetabolism in several areas of the brain that is unchanged over a mean of 14.9 months, which may represent a resting metabolic signature for intrinsic SLE-associated pathology that correlates with CD. The associated DTI studies identified WM clusters with reduced microstructural integrity that were connected by significantly diminished tracts, several of which were adjacent to the hypermetabolic regions. Moreover, relationships among serum DNRAb, hippocampal glucose metabolism, microstructural integrity in the parahippocampus, and performance on spatial memory tasks suggest that an initial insult occurring in gray matter may lead to decreased integrity of WM outflow tracts, resulting in impaired memory function. In this case it may be DNRAb that is the inciting factor but many other inflammatory molecules, including IFN- $\alpha$, IFN- $\gamma$, IL-1 $\beta$, IL-6, and others have been implicated $(10,12,13)$. Continued longitudinal studies are necessary to prove our hypothesis as are other types of imaging studies to determine the contributions of neuronal versus microglial cell activity to regional hypermetabolism. To avoid confounding influences on our cognitive, behavioral, and imaging outcomes, we have restricted our subject population; however, it will also be useful to validate our findings in SLE patients with a broader spectrum of disease activity to see if changes in disease activity or medications alter the resting metabolic signature.

\section{Methods}

\section{Study design}

This is a prospective, observational study designed to evaluate associations among brain regional resting metabolism, microstructural integrity, serum DNRAb titers, and performance on cognitive testing in SLE subjects with quiescent disease and no history of CNS insult or known neuropsychological illness. Assessments were performed at baseline and after a mean of 15 months in the SLE subjects. Imaging studies from SLE subjects were compared with imaging studies from age- and sex-matched historical healthy control subjects.

\section{Subject selection}

SLE subjects in groups 1 and 2. Thirty-seven SLE subjects were recruited from the Rheumatology Clinics at the Feinstein Institute for Medical Research (FIMR). All were 18 years of age or older and fulfilled American College of Rheumatology revised criteria for SLE (53). FDG-PET imaging data from the first 17 SLE subjects (group 1; SLE-1) were published previously (16). Data from the newly recruited 20 SLE subjects (group 2; SLE-2), studied under the same FDG-PET imaging protocol as SLE-1, were used separately and in combination with those from group 1 for the validation studies. In addition to baseline FDG-PET imaging, SLE-2 subjects also had MRI-DTI at baseline and repeat PET and DTI studies a mean of 14.9 (range 11.3-19.1 months) months later. To avoid confounding influences on neuroimaging and neuropsychological endpoints, key exclusion criteria included the presence of active or prior NPSLE or other CNS event; structural abnormalities on MRI; current use of antidepressant, antipsychotic, or anxiolytic drugs; or history of illicit drug use. Additionally, SLE subjects were required to have stable disease activity and 
medication doses for 4 weeks prior to assessments. Group 1 recruitment was also stratified by disease duration, with presence of disease for less than 2 or more than 10 years. As disease duration did not contribute to regional brain hypermetabolism (1), group 2 subjects were not stratified by disease duration.

Healthy control subjects. For FDG-PET studies, we studied 25 healthy control (HC-1/2) subjects, including the $17 \mathrm{HC}$ subjects (HC-1) in the previously published study of the SLE-1 subjects (16) and 8 HC subjects (HC-2; 2 newly recruited and 6 selected historical HCs at the Center for Neurosciences in the FIMR), matched for sex and age to the SLE-2 subjects. For MRI-DTI studies, 14 age- and sex-matched healthy controls (HC-3) were selected from a historical cohort of diffusion MRI studies at the FIMR. As the majority of $\mathrm{HC}$ subjects (23 of 25) were selected from an imaging historical healthy control cohort established at the Center for Neurosciences in the FIMR, we were able to match for sex and age but not for ethnicity, race, BMI, and socioeconomic status even though race, BMI, and socioeconomic status have been reported to influence structural brain development $(54,55)$. The HC subjects also did not perform the cognitive and behavioral testing.

\section{Assessments}

Clinical, cognitive, and behavioral assessments for SLE subjects. Disease activity and accrued damage were assessed with the Safety of Estrogens in Lupus Erythematosus National Assessment-SLE Disease Activity Index (SELENA-SLEDAI) (56) and the Systemic Lupus International Collaborating Clinics Damage Index (SLICC DI) (57) within 2 weeks of imaging and cognitive assessments. CD was assessed with the ANAM (58), a computerized battery of measures of sustained attention, visual search, computational skills, concentration, cognitive processing, and working memory. The following ANAM subtests were selected for this study the Matching Grids test, a measure of spatial processing efficiency, where two designs are shown sequentially and the viewer is asked to determine if they are the same as or different from each other; the Match-to-Sample test, a measure of visuospatial perception and working memory, where the subject is asked to select which of two checkerboard matrix designs match a target checkerboard matrix design presented 5 seconds earlier; and the CPT, a measure of vigilance and sustained attention where a running set of numbers is presented in a randomized sequence. For this test, the subject is asked to continuously monitor the numbers and determine if each new number is the same as or different from the preceding number (59).

Throughput scores for each ANAM subtest, representing a combination of reaction time and accuracy, were the primary measure of cognitive efficiency used in the analyses. As data for the majority of HC subjects was obtained from a historical healthy control cohort, they did not undergo cognitive and behavioral testing. Normative data from community and college subjects was used for comparison with the SLE subject data. We did not assign a strict cutoff for cognitive impairment but report the percentages of SLE subjects scoring less than the normative mean or 2 SDs below the normative mean. The raw throughput scores for the ANAM tests were used in the analyses. SLE subjects were rated for depression and anxiety with the BDI $(18,60)$, STAI (19), and the ANAM mood domain assessments. The 2x2SMT assessed both object recognition and memory for spatial relations (Supplemental Figure 1) (15). All assessments were completed at baseline and repeated in 13 of the SLE-2 subjects after a mean of 14.9 months. Four of the SLE- 2 subjects withdrew from the longitudinal study, and all of the remaining 16 SLE-2 subjects underwent follow-up assessments; however, both the research MRI and PET scanners at the institution were replaced during that time. To alleviate concerns associated with analyses of data derived from different scanners, only follow-up data for 13 SLE subjects with repeat studies on the original scanners are presented here.

PET. At baseline, all 37 SLE (20 SLE-2 and 17 SLE-1) and 25 HC-1/2 subjects (men/women: 2/23, age $40.7 \pm 12.9$ years) underwent FDG-PET imaging following an overnight fast, as described elsewhere (61). Thirteen of the twenty SLE-2 subjects had repeat FDG-PET scans on the same scanner 11-19 months from baseline (mean 14.9 months). Scanning was conducted in 3D mode using the GE Advance tomograph (General Electric Medical Systems) at the FIMR. Autoradiographic PET images were acquired for 20 minutes beginning 35 minutes after the injection of $3.5 \mathrm{mCi}$ FDG in an eyes-open rest state in a dimly lit room with minimal auditory stimulation. FDG-PET images were preprocessed using Statistical Parametric Mapping (SPM5, https://www.fil.ion.ucl.ac.uk/spm/software/spm5; Institute of Neurology, London, United Kingdom) running in MATLAB (MathWorks), as described previously (16, 62). Baseline and repeat scans from the 13 SLE-2 subjects were realigned. Individual images of all SLE and HC subjects were normalized and nonlinearly warped into the Talairach space using a standard PET template and then smoothed with an isotropic Gaussian kernel $(10 \mathrm{~mm})$ in all directions to improve the signal- to-noise ratio. Investigators were blinded to subject identity and clinical status during data acquisition and analysis. 
MRI-DTI. Nineteen of the twenty SLE-2 subjects (men/women: 2/17, age $45.12 \pm 10.58$ years) and 14 HC-3 subjects (men/women: 2/12, age $42.02 \pm 10.72$ years) underwent MRI in the Signa HDxt 3.0 $\mathrm{T}$ scanner (GE Healthcare) at North Shore University Hospital, with an 8-channel head coil. Thirteen (men/women: $0 / 13$, age $43.91 \pm 9.73$ years) of the nineteen SLE-2 subjects had repeat scans a mean of 14.9 months later. For DTI, a single-shot spin-echo echo planar imaging sequence was used with 33 diffusion gradient directions and $5 \mathrm{~B} 0$ images. The $\mathrm{b}$ value in the diffusion-weighted images was $800 \mathrm{~s} / \mathrm{mm}^{2}$. The FOV was $240 \mathrm{~mm}$, 55 slices were acquired with $2.5-\mathrm{mm}$ thickness, TE $=82.7 \mathrm{~ms}$, TR $=15$ seconds, flip angle $=90^{\circ}$, and scan time $=9.5 \mathrm{~min}$. The $128 \times 128$ images were 0 filled to a matrix size $256 \times 256$, yielding an image resolution of $0.9 \times 0.9 \times 2.5 \mathrm{~mm}^{3}$. For high-resolution T1-weighted structural scans, the FOV was $240 \mathrm{~mm}, 176$ slices were acquired with 1 -mm thickness, the imaging matrix was $256 \times 256$, flip angle $=8^{\circ}, \mathrm{TR}=7.6 \mathrm{~ms}, \mathrm{TE}=2.9 \mathrm{~ms}$, inversion time $=650 \mathrm{~ms}$, with resolution of $0.9 \times 0.9 \times 1 \mathrm{~mm}^{3}$. After DTI acquisition, the following DTI processing steps were performed using the FMRIB library (http:// www.fmrib.ox.ac.uk/fsl/). Diffusion-weighted images were corrected for eddy current distortions and head motion using the eddy current correction routine in (EDDY) (63). Nonbrain tissue was removed from the images using the brain extraction tool (BET2). Diffusion tensor for each brain voxel and FA map were determined for all subjects using DTIFIT. B0 image was registered to the Montreal Neurological Institute (MNI-152: $1 \times 1 \times 1 \mathrm{~mm}^{3}$ ) template using a 12-parameter affine transformation (FLIRT) (64). The resulting transformation was then applied to the FA map to register it to MNI space. Once aligned, the FA images were smoothed using a kernel of $8 \mathrm{~mm}$ (FWHM) for group comparison and regression analysis.

FA was chosen specifically as the optimal measure for WM structural integrity, as it reflects WM fiber density, axonal diameter, and myelination. Four commonly used measures of DTI include FA, mean diffusivity (MD), axial diffusivity $(\mathrm{AD})$, and radial diffusivity $(\mathrm{RD})(65)$. These measures directly relate to the value of the 3 eigen values $\left(\lambda_{1}, \lambda_{2}, \lambda_{3}\right)$ of the tensor, with different individual properties. MD is a measure preferentially used to probe gray matter microstructure because gray matter tissue lacks directional diffusion, whereas FA, RD, and $\mathrm{AD}$ are used to assess WM microstructure due to inherent directional diffusivity present in WM (66). Although $\mathrm{AD}$ is more specific for axonal degeneration and $\mathrm{RD}$ is more specific for demyelination, FA is highly sensitive to microstructural WM changes (65) and was therefore selected to assess the WM changes in SLE patients in this paper.

\section{Statistics}

Baseline characteristics of SLE subjects and ANAM throughput scores were analyzed using Student's $t$ tests or nonparametric Mann-Whitney $U$ tests, $\chi^{2}$ tests, or Fisher's exact test, as appropriate. Relationships among resting regional metabolism, ANAM scores, and clinical parameters were examined using Pearson's correlations. Differences between T1 and T2 for the longitudinal studies were analyzed with paired $t$ tests or the Exact McNemar test. Analyses were performed using SAS9.4.

FDG-PET analysis. To prospectively validate the significant findings from group 1 (1), metabolic activity in each previously identified hypermetabolic region was measured for group 2 subjects (SLE-2 and HC-2) using spherical (radius $=5 \mathrm{~mm}$ ) volumes of interest (VOIs), centered at the peak voxel of each cluster and normalized by dividing the global metabolic rate measured in each scan. To further assess abnormal regional metabolism in the SLE-2 subjects, a voxel-wise search of whole-brain volume using the SPM5 2-sample $t$ test option was performed to compare scans from the 20 SLE-2 subjects and the $25 \mathrm{HC}-1 / 2$. Moreover, nearly identical subject selection protocols for SLE-1 and SLE-2 allowed us to combine the 37 SLE-1/2 scans and compare them with the $25 \mathrm{HC}-1 / 2$ scans in another voxel-wise search of whole-brain volume using the SPM5 2-sample $t$ test option. For significant regions identified in each SPM analysis, VOIs were used to measure regional metabolism in each of the SLE-1/2 and HC-1/2 subjects. For both analyses, SPM ( $\mathrm{T}$ ) maps were created and considered significant at the threshold of $\mathrm{T} \geq 3.2, P<0.001$ (peak voxel, uncorrected). Regional metabolism was compared between the SLE-1/2 and HC-1/2 subjects or between the SLE-1 and SLE-2 subjects using Student's $t$ tests in post hoc statistical analyses following the SPM imaging analysis. Longitudinal changes in regional metabolism in the SLE-2 subjects were compared between baseline and follow-up (mean 14.9 months) using paired Student's $t$ tests.

DTI analyses - group differences. Voxel-wise searches for microstructural differences between SLE-2 and HC-3 groups at baseline were conducted using SPM8 software (http://www.fil.ion.ucl.ac.uk/spm/ software/spm8/). A WM mask in SPM template was applied in this analysis. The voxel-wise FA group contrasts were considered significant at $P=0.001$ at peak voxel, corrected for cluster extent at $P<0.05$. We 
also reported regional differences at a hypothesis-testing threshold of $P<0.001$ (voxel level uncorrected), with a cluster cutoff of 100 voxels. Individual FA values from each significant cluster were computed for each subject at the baseline and follow-up scans. The individual subject data were inspected for outliers and overlapping values between the groups. Group differences were evaluated post hoc using Student's $t$ test. Microstructural integrity changes over time were evaluated post hoc using paired Student's $t$ tests.

Tractography. Based on the assumption that the orientation of the longest axis (v1) of the diffusion tensor represents local fiber orientation (67), 3D streamlined information from the tensor field, called tractography, were reconstructed. This tractography method requires seeds from which streamlines are propagated based on v1 orientations. The streamlines are terminated when they reach a low anisotropy region where there is no coherent fiber organization. Voxel-based comparison of the FA maps for SLE-2 compared with HC-3 subjects identified clusters with significantly reduced microstructural integrity that were used as seed volumes for tractography. WM pathways passing through these clusters were reconstructed separately for the two groups. The main purpose of showing these group fiber tracts was to visualize WM trajectories in 3 dimensions, particularly in relation to areas with abnormal microstructure (local FA) in the SLE brain. This allowed us to identify the WM pathways associated with these abnormal clusters and to evaluate potential disruption of anatomical connectivity in the SLE group occurring in the vicinity of these clusters. Diffusion-weighted images from subjects in each group were registered to the template; the gradient vectors were reoriented for tensor calculation $(68,69)$; and TrackVis (Massachusetts General Hospital; http:// www.trackvis.org/) was used to reconstruct and visualize the group tractography. The fiber-tracking program (Diffusion Toolkit) takes tensor files and generates whole-brain fiber tracks saved to a track data file. The visualization program (TrackVis) utilizes the track data file to visualize the tracks. Tracking parameters were identical for the two groups. The WM mask, the angle threshold of 35, and z-inversion were set up in TrackVis. The reconstructed tracts were displayed for SLE-2 and HC-3.

Spatial memory test correlation. Voxel-wise correlations between FA maps and serum DNRAb titers in SLE-2 subjects were assessed using regression analysis in SPM8 software. The voxel-wise contrasts were considered significant at a hypothesis-testing threshold of $P<0.001$ (voxel level uncorrected), with a cluster cutoff of 100 voxels. Individual FA values from each significant cluster were computed for each subject. Correlations between these FA values, serum DNRAb titers, and 2x2SMT scores were evaluated post hoc using Pearson's product-moment correlation coefficient.

All statistical tests in this study were considered significant at $P<0.05$ and were 2 tailed.

\section{Study approval}

The research was in compliance with the Helsinki Declaration and approved by the Institutional Review Board and the Institutional Radioactive Dose Research Committee of the Northwell Health System. Informed consent was obtained prior to all study procedures.

\section{Author contributions}

$\mathrm{MM}, \mathrm{BD}, \mathrm{DE}, \mathrm{BTV}$, and CA participated in study conception and design, data interpretation, and drafting the manuscript. AV, CCT, and MS participated in data analysis and interpretation and drafting the manuscript. EJP, EWA, JS, BB, SK, PW, and CS participated in data acquisition, interpretation of the data, and drafting the manuscript. All authors gave final approval of the submitted manuscript.

\section{Acknowledgments}

This work was supported by a grant from the National Institute of Allergy and Infectious Diseases at the National Institutes of Health (research grant 1P01AI073693). The authors would like to thank all of the patients and healthy individuals who contributed to this study. They would also like to thank Andrew Shaw for his dedication to this study, help with subject recruitment and scheduling, and overall support.

Address correspondence to: Meggan Mackay, Feinstein Institute for Medical Research, Northwell Health Manhasset, New York 11030, USA. Phone: 516.562.3838; Email: mmackay@northwell.edu.

1. Ainiala H, Loukkola J, Peltola J, Korpela M, Hietaharju A. The prevalence of neuropsychiatric syndromes in systemic lupus erythematosus. Neurology. 2001;57(3):496-500.

2. Brey RL, et al. Neuropsychiatric syndromes in lupus: prevalence using standardized definitions. Neurology. 2002;58(8):1214-1220. 
3. Appenzeller S, Cendes F, Costallat LT. Cognitive impairment and employment status in systemic lupus erythematosus: a prospective longitudinal study. Arthritis Rheum. 2009;61(5):680-687.

4. Calderón J, et al. Impact of cognitive impairment, depression, disease activity, and disease damage on quality of life in women with systemic lupus erythematosus. Scand J Rheumatol. 2017;46(4):273-280.

5. Jönsen A, Bengtsson AA, Nived O, Ryberg B, Sturfelt G. Outcome of neuropsychiatric systemic lupus erythematosus within a defined Swedish population: increased morbidity but low mortality. Rheumatology (Oxford). 2002;41(11):1308-1312.

6. Leslie B, Crowe SF. Cognitive functioning in systemic lupus erythematosus: a meta-analysis. Lupus. 2018;27(6):920-929.

7. Rayes HA, et al. What is the prevalence of cognitive impairment in lupus and which instruments are used to measure it? A systematic review and meta-analysis. Semin Arthritis Rheum. 2018;48(2):240-255.

8. DeGiorgio LA, Konstantinov KN, Lee SC, Hardin JA, Volpe BT, Diamond B. A subset of lupus anti-DNA antibodies cross-reacts with the NR2 glutamate receptor in systemic lupus erythematosus. Nat Med. 2001;7(11):1189-1193.

9. Mader S, Brimberg L, Diamond B. The role of brain-reactive autoantibodies in brain pathology and cognitive impairment. Front Immunol. 2017;8:1101

10. Bialas AR, et al. Microglia-dependent synapse loss in type I interferon-mediated lupus. Nature. 2017;546(7659):539-543.

11. González A, Massardo L. Antibodies and the brain: antiribosomal P protein antibody and the clinical effects in patients with systemic lupus erythematosus. Curr Opin Neurol. 2018;31(3):300-305.

12. Mackay M. Lupus brain fog: a biologic perspective on cognitive impairment, depression, and fatigue in systemic lupus erythematosus. Immunol Res. 2015;63(1-3):26-37.

13. Postal M, et al. Interferon- $\gamma$ is associated with cerebral atrophy in systemic lupus erythematosus. Neuroimmunomodulation. 2017;24(2):100-105.

14. Faust TW, et al. Neurotoxic lupus autoantibodies alter brain function through two distinct mechanisms. Proc Natl Acad Sci USA. 2010;107(43):18569-18574.

15. Chang EH, et al. Selective impairment of spatial cognition caused by autoantibodies to the N-Methyl-D-Aspartate receptor. EBioMedicine. 2015;2(7):755-764.

16. Mackay M, et al. Brain metabolism and autoantibody titres predict functional impairment in systemic lupus erythematosus. Lupus Sci Med. 2015;2(1):e000074.

17. Costallat BL, Ferreira DM, Lapa AT, Rittner L, Costallat LTL, Appenzeller S. Brain diffusion tensor MRI in systematic lupus erythematosus: A systematic review. Autoimmun Rev. 2018;17(1):36-43.

18. Beck AT, Steer RA, and Brown GK. Manual for the Beck Depression Inventory-II. San Antonio, TX: Harcourt Brace \& Company; 1996.

19. Julian LJ. Measures of anxiety: State-Trait Anxiety Inventory (STAI), Beck Anxiety Inventory (BAI), and Hospital Anxiety and Depression Scale-Anxiety (HADS-A). Arthritis Care Res (Hoboken). 2011;63 Suppl 11:S467-S472.

20. Muscal E, Brey RL. Neurologic manifestations of systemic lupus erythematosus in children and adults. Neurol Clin. 2010;28(1):61-73.

21. Curiel R, Akin EA, Beaulieu G, DePalma L, Hashefi M. PET/CT imaging in systemic lupus erythematosus. Ann N Y Acad Sci. 2011;1228:71-80.

22. Komatsu N, et al. Decreased regional cerebral metabolic rate for glucose in systemic lupus erythematosus patients with psychiatric symptoms. Eur Neurol. 1999;42(1):41-48.

23. Lee SW, Park MC, Lee SK, Park YB. The efficacy of brain (18)F-fluorodeoxyglucose positron emission tomography in neuropsychiatric lupus patients with normal brain magnetic resonance imaging findings. Lupus. 2012;21(14):1531-1537.

24. Saito T, et al. Regional cerebral glucose metabolism in systemic lupus erythematosus patients with major depressive disorder. $J$ Neurol Sci. 2017;379:127-130.

25. Weiner SM, et al. Diagnosis and monitoring of central nervous system involvement in systemic lupus erythematosus: value of F-18 fluorodeoxyglucose PET. Ann Rheum Dis. 2000;59(5):377-385.

26. Krakauer M, Law I. FDG PET brain imaging in neuropsychiatric systemic lupus erythematosis with choreic symptoms. Clin Nucl Med. 2009;34(2):122-123.

27. Niu N, Cui R. Glucose Hypermetabolism in contralateral basal ganglia demonstrated by serial FDG PET/CT scans in a patient with SLE chorea. Clin Nucl Med. 2017;42(1):64-65.

28. Brendel M, et al. Time Courses of Cortical Glucose Metabolism and Microglial Activity Across the Life Span of Wild-Type Mice: A PET Study. J Nucl Med. 2017;58(12):1984-1990.

29. Fan Z, et al. Influence of microglial activation on neuronal function in Alzheimer's and Parkinson's disease dementia. Alzheimers Dement. 2015;11(6):608-21.e7.

30. Mergenthaler P, Lindauer U, Dienel GA, Meisel A. Sugar for the brain: the role of glucose in physiological and pathological brain function. Trends Neurosci. 2013;36(10):587-597.

31. Patel AB, et al. Direct evidence for activity-dependent glucose phosphorylation in neurons with implications for the astrocyte-to-neuron lactate shuttle. Proc Natl Acad Sci USA. 2014;111(14):5385-5390.

32. Vo A, et al. Regional brain metabolism in a murine systemic lupus erythematosus model. J Cereb Blood Flow Metab. 2014;34(8):1315-1320.

33. Jung RE, et al. Diffusion tensor imaging in neuropsychiatric systemic lupus erythematosus. BMC Neurol. 2010;10:65.

34. Lee SP, Wu CS, Cheng JZ, Chen CM, Chen YC, Chou MC. Automatic Segmentation of the Corpus Callosum Using a Cell-Competition Algorithm: Diffusion Tensor Imaging-Based Evaluation of Callosal Atrophy and Tissue Alterations in Patients With Systemic Lupus Erythematosus. J Comput Assist Tomogr. 2015;39(5):781-786.

35. Schmidt-Wilcke T, et al. Diminished white matter integrity in patients with systemic lupus erythematosus. Neuroimage Clin. 2014;5:291-297.

36. Zhang L, et al. Diffusion changes in patients with systemic lupus erythematosus. Magn Reson Imaging. 2007;25(3):399-405

37. Zimny A, et al. In vivo evaluation of brain damage in the course of systemic lupus erythematosus using magnetic resonance spectroscopy, perfusion-weighted and diffusion-tensor imaging. Lupus. 2014;23(1):10-19.

38. Cannerfelt B, et al. White matter lesions and brain atrophy in systemic lupus erythematosus patients: correlation to cognitive dysfunction in a cohort of systemic lupus erythematosus patients using different definition models for neuropsychiatric systemic 
lupus erythematosus. Lupus. 2018;27(7):1140-1149.

39. Nystedt J, et al. Altered white matter microstructure in lupus patients: a diffusion tensor imaging study. Arthritis Res Ther. 2018;20(1):21.

40. Shapira-Lichter I, et al. Impaired diffusion tensor imaging findings in the corpus callosum and cingulum may underlie impaired learning and memory abilities in systemic lupus erythematosus. Lupus. 2016;25(11):1200-1208.

41. Xu M, et al. Alterations of white matter structural networks in patients with non-neuropsychiatric systemic lupus erythematosus identified by probabilistic tractography and connectivity-based analyses. Neuroimage Clin. 2017;13:349-360.

42. Wiseman SJ, et al. Fatigue and cognitive function in systemic lupus erythematosus: associations with white matter microstructural damage. A diffusion tensor MRI study and meta-analysis. Lupus. 2017;26(6):588-597.

43. Reagh ZM, Ranganath C. What does the functional organization of cortico-hippocampal networks tell us about the functional organization of memory? Neurosci Lett. 2018;680:69-76.

44. Appenzeller S, Carnevalle AD, Li LM, Costallat LT, Cendes F. Hippocampal atrophy in systemic lupus erythematosus. Ann Rheum Dis. 2006;65(12):1585-1589.

45. Bódi N, Polgár A, Kiss E, Mester Á, Poór G, Kéri S. Reduced volumes of the CA1 and CA4-dentate gyrus hippocampal subfields in systemic lupus erythematosus. Lupus. 2017;26(13):1378-1382.

46. Brunner R, Schaefer D, Hess K, Parzer P, Resch F, Schwab S. Effect of corticosteroids on short-term and long-term memory. Neurology. 2005;64(2):335-337.

47. Kozora E, et al. Memory impairment associated with neurometabolic abnormalities of the hippocampus in patients with non-neuropsychiatric systemic lupus erythematosus. Lupus. 2011;20(6):598-606.

48. Zimmermann N, et al. Global Cognitive Impairment in Systemic Lupus Erythematosus Patients: A Structural MRI Study. Clin Neuroradiol. 2017;27(1):23-29.

49. Lauvsnes MB, et al. Association of hippocampal atrophy with cerebrospinal fluid antibodies against the NR2 subtype of the $\mathrm{N}$-methyl-D-aspartate receptor in patients with systemic lupus erythematosus and patients with primary Sjogren's syndrome. Arthritis Rheumatol. 2014;66(12):3387-3394.

50. Ballok DA, Woulfe J, Sur M, Cyr M, Sakic B. Hippocampal damage in mouse and human forms of systemic autoimmune disease. Hippocampus. 2004;14(5):649-661.

51. Sakic B, Kolb B, Whishaw IQ, Gorny G, Szechtman H, Denburg JA. Immunosuppression prevents neuronal atrophy in lupusprone mice: evidence for brain damage induced by autoimmune disease? J Neuroimmunol. 2000;111(1-2):93-101.

52. Kowal C, et al. Cognition and immunity; antibody impairs memory. Immunity. 2004;21(2):179-188.

53. Hochberg MC. Updating the American College of Rheumatology revised criteria for the classification of systemic lupus erythematosus. Arthritis Rheum. 1997;40(9):1725.

54. Chao SH, et al. Correlation between brain circuit segregation and obesity. Behav Brain Res. 2018;337:218-227.

55. Brito NH, Noble KG. Socioeconomic status and structural brain development. Front Neurosci. 2014;8:276.

56. Petri M, et al. Combined oral contraceptives in women with systemic lupus erythematosus. NEngl J Med. 2005;353(24):2550-2558.

57. Gladman DD, et al. The reliability of the Systemic Lupus International Collaborating Clinics/American College of Rheumatology Damage Index in patients with systemic lupus erythematosus. Arthritis Rheum. 1997;40(5):809-813.

58. Roebuck-Spencer TM, et al. Use of computerized assessment to predict neuropsychological functioning and emotional distress in patients with systemic lupus erythematosus. Arthritis Rheum. 2006;55(3):434-441.

59. Bleiberg J, Kane RL, Reeves DL, Garmoe WS, Halpern E. 2000. Factor analysis of computerized and traditional tests in mild brain injury research. Clin Neuropsychol. 2000;14(3):287-94.

60. Beck AT, Ward CH, Mendelson M, Mock J, Erbaugh J. An inventory for measuring depression. Arch Gen Psychiatry. 1961;4:561-571.

61. Ma Y, Tang C, Spetsieris PG, Dhawan V, Eidelberg D. Abnormal metabolic network activity in Parkinson's disease: test-retest reproducibility. J Cereb Blood Flow Metab. 2007;27(3):597-605.

62. Spetsieris PG, Eidelberg D. Scaled subprofile modeling of resting state imaging data in Parkinson's disease: methodological issues. Neuroimage. 2011;54(4):2899-2914.

63. Andersson JLR, Sotiropoulos SN. An integrated approach to correction for off-resonance effects and subject movement in diffusion MR imaging. Neuroimage. 2016;125:1063-1078.

64. Jenkinson M, Bannister P, Brady M, Smith S. Improved optimization for the robust and accurate linear registration and motion correction of brain images. Neuroimage. 2002;17(2):825-841.

65. Alexander AL, Lee JE, Lazar M, Field AS. Diffusion tensor imaging of the brain. Neurotherapeutics. 2007;4(3):316-329.

66. Feldman HM, Yeatman JD, Lee ES, Barde LH, Gaman-Bean S. Diffusion tensor imaging: a review for pediatric researchers and clinicians. J Dev Behav Pediatr. 2010;31(4):346-356.

67. Mori S, Zhang J. Principles of diffusion tensor imaging and its applications to basic neuroscience research. Neuron. 2006;51(5):527-539

68. Vo A, Argyelan M, Eidelberg D, Uluğ AM. Early registration of diffusion tensor images for group tractography of dystonia patients. J Magn Reson Imaging. 2013;37(1):67-75.

69. Vo A, et al. Thalamocortical connectivity correlates with phenotypic variability in dystonia. Cereb Cortex. 2015;25(9):3086-3094. 